\title{
Ab Initio Study of the Addition - Fragmentation Equilibrium in RAFT Polymerization: When is Polymerization Retarded?
}

Michelle L. Coote*

Research School of Chemistry, Australian National University, Canberra, ACT 0200, Australia

\section{SUPPORTING INFORMATION}

(Tables S1, Total 14 pages)

\footnotetext{
* To whom correspondence should be addressed, email: mcoote@rsc.anu.edu.au
} 
Table S1. GAUSSIAN Archive Entries for B3-LYP/6-31G(d) Optimized Geometries

\section{$\mathrm{CH}_{3} \mathrm{SC} \cdot\left(\mathrm{CH}_{3}\right) \mathrm{S}-\mathrm{CH}_{3}(1)$}

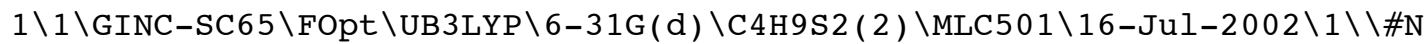
UB3LYP $/ 6-31 \mathrm{G}(\mathrm{D})$ FOPT $=(\mathrm{Z}-\mathrm{MATRIX}, \mathrm{TIGHT}, \mathrm{MAXCYC}=100)$ FREQ MAXDISK $=13107200$ $0 \mathrm{SCF}=(\mathrm{TIGHT}, \mathrm{MAXCYC}=100) \mathrm{GEOM}=\mathrm{CHECK}$ GUESS $=$ READ $\backslash \backslash$ mescmesme $-\mathrm{c} 1 \mathrm{~b}-6 \mathrm{dub} 3 \backslash \backslash 0$ , $2 \backslash \mathrm{H} \backslash \mathrm{C}, 1, \mathrm{~B} 1 \backslash \mathrm{H}, 2$, $\mathrm{B} 2,1, \mathrm{~A} 1 \backslash \mathrm{S}, 2, \mathrm{~B} 3,1, \mathrm{~A} 2,3, \mathrm{D} 1,0 \backslash \mathrm{C}, 4, \mathrm{~B} 4,2, \mathrm{~A} 3,1, \mathrm{D} 2,0 \backslash \mathrm{S}, 5, \mathrm{~B} 5,4$ , $\mathrm{A} 4,2, \mathrm{D} 3,0 \backslash \mathrm{C}, 6, \mathrm{~B} 6,5, \mathrm{~A} 5,4,-\mathrm{D} 4,0 \backslash \mathrm{H}, 7, \mathrm{~B} 7,6, \mathrm{~A} 6,5, \mathrm{D} 5,0 \backslash \mathrm{H}, 7, \mathrm{~B} 8,6, \mathrm{~A} 7,8, \mathrm{D} 6,0 \backslash \mathrm{H}$ , $7, \mathrm{~B} 9,6, \mathrm{~A} 8,8, \mathrm{D} 7,0 \backslash \mathrm{C}, 5, \mathrm{~B} 10,4, \mathrm{~A} 9,2, \mathrm{D} 8,0 \backslash \mathrm{H}, 2, \mathrm{~B} 11,1, \mathrm{~A} 10,3, \mathrm{D} 9,0 \backslash \mathrm{H}, 11, \mathrm{~B} 12,5$, $\mathrm{A} 11,4, \mathrm{D} 10,0 \backslash \mathrm{H}, 11, \mathrm{~B} 13,5, \mathrm{~A} 12,4, \mathrm{D} 11,0 \backslash \mathrm{H}, 11, \mathrm{~B} 14,5, \mathrm{~A} 13,4, \mathrm{D} 12,0 \backslash \backslash \mathrm{B} 1=1.092657$ $93 \backslash \mathrm{B} 2=1.09285939 \backslash \mathrm{B} 3=1.83510395 \backslash \mathrm{B} 4=1.77013049 \backslash \mathrm{B} 5=1.75804982 \backslash \mathrm{B} 6=1.832220$ $08 \backslash B 7=1.09257315 \backslash B 8=1.09085787 \backslash B 9=1.09328827 \backslash B 10=1.50447696 \backslash B 11=1.0934$ $6331 \backslash \mathrm{B} 12=1.09613505 \backslash \mathrm{B} 13=1.09553397 \backslash \mathrm{B} 14=1.10178689 \backslash \mathrm{A} 1=110.0297286 \backslash \mathrm{A} 2=11$ $0.82443615 \backslash A 3=102.23773298 \backslash A 4=118.51568336 \backslash A 5=103.96848806 \backslash A 6=110.4950$ $1693 \backslash A 7=111.21266523 \backslash A 8=105.91951214 \backslash A 9=120.43443778 \backslash A 10=109.33705389 \backslash$ $\mathrm{A} 11=111.15729093 \backslash \mathrm{A} 12=110.74166016 \backslash \mathrm{A} 13=112.20742397 \backslash \mathrm{D} 1=-124.06148497 \backslash \mathrm{D} 2$ $=60.56470818 \backslash \mathrm{D} 3=-144.49451711 \backslash \mathrm{D} 4=-48.977946 \backslash \mathrm{D} 5=60.52936234 \backslash \mathrm{D} 6=-122.478$ $49479 \backslash D 7=118.55770463 \backslash D 8=62.40591497 \backslash D 9=119.28641302 \backslash D 10=-44.11186517 \backslash$ $\mathrm{D} 11=-164.00422423 \backslash \mathrm{D} 12=75.46393503 \backslash \backslash$ Version=DEC-AXP-OSF / 1-G98RevA.11.3\ $\mathrm{HF}=-954.1760596 \backslash \mathrm{S} 2=0.755169 \backslash \mathrm{S} 2-1=0 . \backslash \mathrm{S} 2 \mathrm{~A}=0.75002 \backslash \mathrm{RMSD}=5.132 \mathrm{e}-09 \backslash \mathrm{RMSF}=2$. $815 e-05 \backslash \mathrm{Dipole}=0.2098476,0.5512682,-0.4657697 \backslash \mathrm{PG}=\mathrm{C} 01 \quad[\mathrm{X}(\mathrm{C} 4 \mathrm{H} 9 \mathrm{~S} 2)] \backslash \backslash @$

\section{$\mathrm{CH}_{3} \mathrm{SC} \cdot(\mathrm{Ph}) \mathrm{S}-\mathrm{CH}_{3}(2)$}

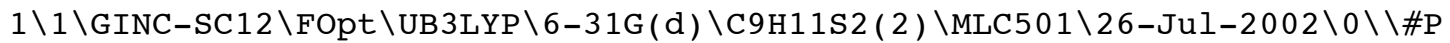
B3LYP $/ 6-31 \mathrm{G} * \mathrm{OPT}=(\mathrm{MAXCYC}=100) \mathrm{SCF}=(\mathrm{MAXCYC}=100)$ FREQ MAXDISK $=131072000$ $\backslash \backslash \mathrm{rrad} z=\mathrm{ph} r=\mathrm{me}-\mathrm{c} 1 \mathrm{c}-6 \mathrm{duhf}--\mathrm{C} 2 \backslash \backslash 0,2 \backslash \mathrm{C}, 0 ., 0 .,-0.5436243969 \backslash \mathrm{C}, 0 ., 0 ., 0.89$ $993655 \overline{5} 8 \backslash \mathrm{C}, \overline{1} .2045767983,-0.1407684102,1.6447473811 \backslash \mathrm{C},-1.2045767983,0.1$ $407684102,1.6447473811 \backslash \mathrm{C}, 1.1980023223,-0.1372926446,3.0327870849 \backslash \mathrm{C},-1$. $1980023223,0.1372926446,3.0327870849 \backslash \mathrm{H}, 2.1407212827,-0.2659311883,1.11$ $24152303 \backslash \mathrm{H},-2.1407212827,0.2659311883,1.1124152303 \backslash \mathrm{H}, 2.1367874102,-0.2$ $51386417,3.5689285696 \backslash \mathrm{H},-2.1367874102,0.251386417,3.5689285696 \backslash \mathrm{C}, 0 ., 0$.

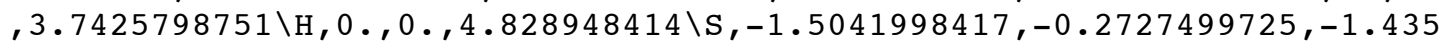
$6947229 \backslash \mathrm{C},-1.5483731257,1.1133863252,-2.639460946 \backslash \mathrm{H},-2.4327150559,0.94$ $51928411,-3.2601525901 \backslash \mathrm{H},-1.6417073267,2.0701943282,-2.120752452 \backslash \mathrm{H},-0$. $656494302,1.1210366726,-3.2689146201 \backslash \mathrm{S}, 1.5041998417,0.2727499725,-1.43$ $56947229 \backslash \mathrm{C}, 1.5483731257,-1.1133863252,-2.639460946 \backslash \mathrm{H}, 2.4327150559,-0.9$ $451928411,-3.2601525901 \backslash \mathrm{H}, 1.6417073267,-2.0701943282,-2.120752452 \backslash \mathrm{H}, 0$. $656494302,-1.1210366726,-3.2689146201 \backslash \backslash$ Version=DEC-AXP-OSF / 1-G98RevA. 1 $1.3 \backslash$ State $=2-B \backslash H F=-1145.9239982 \backslash S 2=0.772251 \backslash \mathrm{S} 2-1=0 . \backslash \mathrm{S} 2 \mathrm{~A}=0.750367 \backslash \mathrm{RMSD}=6$ $.432 \mathrm{e}-09 \backslash \mathrm{RMSF}=1.039 \mathrm{e}-05 \backslash \mathrm{Dipole}=0 ., 0 .,-0.434059 \backslash \mathrm{PG}=\mathrm{C} 02 \quad[\mathrm{C} 2(\mathrm{C} 1 \mathrm{C} 1 \mathrm{C} 1 \mathrm{H} 1), \mathrm{X}($ $\mathrm{C} 6 \mathrm{H} 10 \mathrm{~S} 2) \mathrm{J} \backslash \backslash @$

\section{$\mathrm{CH}_{3} \mathrm{SC} \cdot\left(\mathrm{CH}_{2} \mathrm{Ph}\right) \mathrm{S}-\mathrm{CH}_{3}(3)$}

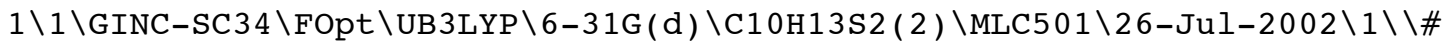

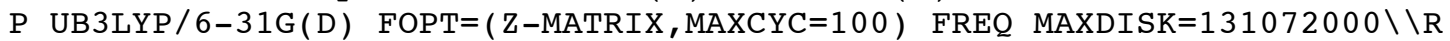
AFT RAD Me-S-C (MePH)-S-Me ( $\mathrm{Z}=\mathrm{MePH} \mathrm{R}=\mathrm{Me})(1 \mathrm{~b}) \backslash \backslash 0,2 \backslash \mathrm{C} \backslash \mathrm{C}, 1, \mathrm{~B} 1 \backslash \mathrm{H}, 2, \mathrm{~B} 2,1, \mathrm{~A} 1$ $\backslash \mathrm{C}, 2, \mathrm{~B} 3,1, \mathrm{~A} 2,3, \mathrm{D} 1,0 \backslash \mathrm{C}, 4, \mathrm{~B} 4,2, \mathrm{~A} 3,1, \mathrm{D} 2,0 \backslash \mathrm{C}, 5, \mathrm{~B} 5,4, \mathrm{~A} 4,2, \mathrm{D} 3,0 \backslash \mathrm{C}, 6, \mathrm{~B} 6,5, \mathrm{~A} 5$, $4, \mathrm{D} 4,0 \backslash \mathrm{C}, 7, \mathrm{~B} 7,6, \mathrm{~A} 6,5, \mathrm{D} 5,0 \backslash \mathrm{C}, 8, \mathrm{~B} 8,7, \mathrm{~A} 7,6, \mathrm{D} 6,0 \backslash \mathrm{H}, 9, \mathrm{~B} 9,8, \mathrm{~A} 8,7, \mathrm{D} 7,0 \backslash \mathrm{H}, 8, \mathrm{~B} 1$ $0,7, \mathrm{~A} 9,9, \mathrm{D} 8,0 \backslash \mathrm{H}, 7, \mathrm{~B} 11,6, \mathrm{~A} 10,8, \mathrm{D} 9,0 \backslash \mathrm{H}, 6, \mathrm{~B} 12,5, \mathrm{~A} 11,7, \mathrm{D} 10,0 \backslash \mathrm{H}, 5, \mathrm{~B} 13,4, \mathrm{~A} 12$ $, 6, \mathrm{D} 11,0 \backslash \mathrm{H}, 2, \mathrm{~B} 14,1, \mathrm{~A} 13,3, \mathrm{D} 12,0 \backslash \mathrm{S}, 1, \mathrm{~B} 15,2, \mathrm{~A} 14,3, \mathrm{D} 13,0 \backslash \mathrm{C}, 16, \mathrm{~B} 16,1, \mathrm{~A} 15,2$, $\mathrm{D} 14,0 \backslash \mathrm{H}, 17, \mathrm{~B} 17,16, \mathrm{~A} 16,1, \mathrm{D} 15,0 \backslash \mathrm{H}, 17, \mathrm{~B} 18,16, \mathrm{~A} 17,18, \mathrm{D} 16,0 \backslash \mathrm{H}, 17, \mathrm{~B} 19,16, \mathrm{~A} 18$ , $18, \mathrm{D} 17,0 \backslash \mathrm{S}, 1, \mathrm{~B} 20,2, \mathrm{~A} 19,16, \mathrm{D} 18,0 \backslash \mathrm{C}, 21, \mathrm{~B} 21,1, \mathrm{~A} 20,2$, D19, 0\H , 22 , B22 , 21 , A2 $1,1, \mathrm{D} 20,0 \backslash \mathrm{H}, 22, \mathrm{~B} 23,21, \mathrm{~A} 22,23, \mathrm{D} 21,0 \backslash \mathrm{H}, 22, \mathrm{~B} 24,21, \mathrm{~A} 23,23, \mathrm{D} 22,0 \backslash \backslash \mathrm{B} 1=1.5157$ $8873 \backslash \mathrm{B} 2=1.09765845 \backslash \mathrm{B} 3=1.52389414 \backslash \mathrm{B} 4=1.40058996 \backslash \mathrm{B} 5=1.39681842 \backslash \mathrm{B} 6=1.3955$ $8668 \backslash \mathrm{B} 7=1.39697813 \backslash \mathrm{B} 8=1.39470243 \backslash \mathrm{B} 9=1.08777803 \backslash \mathrm{B} 10=1.08705661 \backslash \mathrm{B} 11=1.08$ $678408 \backslash \mathrm{B} 12=1.08707699 \backslash \mathrm{B} 13=1.08729883 \backslash \mathrm{B} 14=1.09778413 \backslash \mathrm{B} 15=1.75737506 \backslash \mathrm{B} 16$ $=1.8511538 \backslash \mathrm{B} 17=1.09395743 \backslash \mathrm{B} 18=1.09286269 \backslash \mathrm{B} 19=1.09134704 \backslash \mathrm{B} 20=1.75969536$ 
$\backslash \mathrm{B} 21=1.82647684 \backslash \mathrm{B} 22=1.09192111 \backslash \mathrm{B} 23=1.09332514 \backslash \mathrm{B} 24=1.09120267 \backslash \mathrm{A} 1=108.25$ $525698 \backslash A 2=113.94938997 \backslash A 3=120.79198662 \backslash A 4=120.78731 \backslash A 5=120.13467307 \backslash A 6$ $=119.55192775 \backslash A 7=120.16376976 \backslash A 8=119.72340424 \backslash A 9=120.07880369 \backslash A 10=120$. $24221717 \backslash A 11=119.74087848 \backslash A 12=119.32184346 \backslash A 13=109.03557488 \backslash A 14=120.36$ $439381 \backslash \mathrm{A} 15=104.08221211 \backslash \mathrm{A} 16=106.22727959 \backslash \mathrm{A} 17=110.79627524 \backslash \mathrm{A} 18=110.4665$ $5003 \backslash A 19=115.46042477 \backslash A 20=104.60546169 \backslash A 21=110.57101967 \backslash A 22=105.689005$ $73 \backslash A 23=111.64284706 \backslash D 1=-121.45670912 \backslash D 2=-261.77203284 \backslash D 3=181.36035309 \backslash$ $\mathrm{D} 4=-0.2080065 \backslash \mathrm{D} 5=-0.01087986 \backslash \mathrm{D} 6=0.19371478 \backslash \mathrm{D} 7=-180.12483316 \backslash \mathrm{D} 8=180.168$ $86927 \backslash D 9=180.28334206 \backslash D 10=-179.64263638 \backslash D 11=179.92707274 \backslash D 12=115.75269$ $857 \backslash D 13=49.66353815 \backslash D 14=96.41159877 \backslash D 15=175.79130312 \backslash D 16=-118.67140069$ $\backslash \mathrm{D} 17=119.10764327 \backslash \mathrm{D} 18=170.17863131 \backslash \mathrm{D} 19=166.2284763 \backslash \mathrm{D} 20=-57.73757008 \backslash \mathrm{D} 2$ $1=-118.66229821 \backslash \mathrm{D} 22=122.32870879 \backslash \backslash$ Version=DEC-AXP-OSF $/ 1-G 98 \mathrm{RevA} .11 .3 \backslash \mathrm{H}$ $\mathrm{F}=-1185.2251153 \backslash \mathrm{S} 2=0.755322 \backslash \mathrm{S} 2-1=0 . \backslash \mathrm{S} 2 \mathrm{~A}=0.750022 \backslash \mathrm{RMSD}=2.912 \mathrm{e}-09 \backslash \mathrm{RMSF}=4$ $.358 e-05 \backslash$ Dipole $=-0.3028549,-0.2300317,-0.3558071 \backslash \mathrm{PG}=\mathrm{C} 01 \quad[\mathrm{X}(\mathrm{C} 10 \mathrm{H} 13 \mathrm{~S} 2)] \backslash$ 10

\section{$\mathrm{CH}_{3} \mathrm{SC} \cdot\left(\mathrm{CH}_{3}\right) \mathrm{S}-\mathrm{CH}_{2} \mathrm{Ph}(4)$}

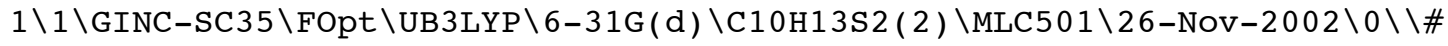
$\mathrm{N}$ UB3LYP/6-31G(D) OPT $=($ MAXCYC $=100)$ FREQ MAXDISK $=131072000$ GUESS=READ G EOM=CHECK $\backslash \backslash$ RAFT-RAD $(\mathrm{Z}=\mathrm{Me} \mathrm{R}=\mathrm{Bz}) \mathrm{c} 1 \mathrm{CY} \backslash \backslash 0,2 \backslash \mathrm{C}, 0.5508217904,1.7349275973,0$ $.082764896 \backslash \mathrm{C}, 0.56372646,1.7473599479,1.5871934348 \backslash \mathrm{S}, 2.1301757851,1.717$ $3441418,-0.6950929289 \backslash \mathrm{C}, 1.7948574291,2.2440328176,-2.4132160553 \backslash \mathrm{S},-0.9$ $429428737,1.4302432185,-0.7838339056 \backslash \mathrm{C},-0.8526164377,-0.3899166038,-1$. $2702362419 \backslash \mathrm{H}, 0.0428795461,-0.5253130123,-1.8826001106 \backslash \mathrm{H},-1.7299201211$, $-0.5085772565,-1.9145364482 \backslash \mathrm{C},-0.875490546,-1.3501477238,-0.1161572952$ $\backslash \mathrm{C},-2.0833512206,-1.7022349815,0.5041883734 \backslash \mathrm{C}, 0.3160949475,-1.90654556$ $57,0.3697099505 \backslash \mathrm{C},-2.1004443834,-2.5879588179,1.5797548248 \backslash \mathrm{C}, 0.3015747$ $185,-2.7911897776,1.4498907745 \backslash \mathrm{C},-0.9065670386,-3.1349590939,2.0577562$ $329 \backslash \mathrm{H},-3.0147042772,-1.2737279948,0.1408925506 \backslash \mathrm{H}, 1.2593017662,-1.64381$ $76211,-0.1034607552 \backslash \mathrm{H},-3.0460141015,-2.8536537482,2.0452370028 \backslash \mathrm{H}, 1.318$ $5665671,3.2267422653,-2.4245626833 \backslash \mathrm{H}, 1.1643996451,1.5261239453,-2.9404$ $378065 \backslash \mathrm{H},-0.9197307406,-3.8264159381,2.8960029584 \backslash \mathrm{H}, 1.2346124547,-3.21$ $48198333,1.8123905538 \backslash \mathrm{H},-0.3169489145,2.2771850403,1.9681460829 \backslash \mathrm{H}, 1.46$ $03717508,2.2435428602,1.9759037853 \backslash \mathrm{H}, 0.5291283588,0.7289034058,2.00268$ $91135 \backslash \mathrm{H}, 2.7706971684,2.3022233323,-2.9027282589 \backslash \backslash V e r s i o n=D E C-A X P-O S F / 1$ -G98RevA. $11.3 \backslash \mathrm{HF}=-1185.2273621 \backslash \mathrm{S} 2=0.755783 \backslash \mathrm{S} 2-1=0 . \backslash \mathrm{S} 2 \mathrm{~A}=0.750026 \backslash \mathrm{RMSD}=6$ $.979 e-09 \backslash \mathrm{RMSF}=3.861 \mathrm{e}-06 \backslash \mathrm{Dipole}=0.0663036,-0.3509731,-0.2514008 \backslash \mathrm{PG}=\mathrm{C} 01$ $[\mathrm{X}(\mathrm{C} 10 \mathrm{H} 13 \mathrm{~S} 2)] \backslash \backslash @$

\section{$\mathrm{CH}_{3} \mathrm{SC} \cdot\left(\mathrm{CH}_{3}\right) \mathrm{S}-\mathrm{CH}_{2} \mathrm{COOCH}_{3}(5)$}

$1 \backslash 1 \backslash G I N C-S C 26 \backslash F O p t \backslash U B 3 L Y P \backslash 6-31 G(d) \backslash C 6 H 1102 S 2$ ( 2) \MLC501\02-Aug-2002 $1 \backslash \backslash$ \# UB3LYP/6-31G* OPT=Z-MATRIX SCF $=(Q C, M A X C Y C=100, T I G H T) \quad F R E Q$ MAXDISK $=13$ $1072000 \backslash \backslash \mathrm{RAFT} R \mathrm{RAD}(\mathrm{Z}=\mathrm{Me} \mathrm{R}=\mathrm{MA}) \mathrm{c} 1 \mathrm{p} / / \mathrm{B} 3 \backslash \backslash 0,2 \backslash \mathrm{S} \backslash \mathrm{C}, 1, \mathrm{~B} 1 \backslash \mathrm{C}, 2, \mathrm{~B} 2,1, \mathrm{~A} 1 \backslash \mathrm{S}, 2, \mathrm{~B} 3$, $1, \mathrm{~A} 2,3, \mathrm{D} 1,0 \backslash \mathrm{H}, 3, \mathrm{~B} 4,2, \mathrm{~A} 3,1, \mathrm{D} 2,0 \backslash \mathrm{H}, 3, \mathrm{~B} 5,2, \mathrm{~A} 4,5, \mathrm{D} 3,0 \backslash \mathrm{H}, 3, \mathrm{~B} 6,2, \mathrm{~A} 5,5, \mathrm{D} 4,0 \backslash \mathrm{C}$ $, 1, \mathrm{~B} 7,2, \mathrm{~A} 6,3, \mathrm{D} 5,0 \backslash \mathrm{C}, 4, \mathrm{~B} 8,2, \mathrm{~A} 7,3, \mathrm{D} 6,0 \backslash \mathrm{H}, 9, \mathrm{~B} 9,4, \mathrm{~A} 8,2, \mathrm{D} 7,0 \backslash \mathrm{H}, 9, \mathrm{~B} 10,4, \mathrm{~A} 9,2$ , $\mathrm{D} 8,0 \backslash \mathrm{C}, 9, \mathrm{~B} 11,4, \mathrm{~A} 10,2$, $\mathrm{D} 9,0 \backslash 0,12, \mathrm{~B} 12,9, \mathrm{~A} 11,4, \mathrm{D} 10,0 \backslash 0,12, \mathrm{~B} 13,9, \mathrm{~A} 12,13, \mathrm{D} 1$ $1,0 \backslash \mathrm{C}, 14, \mathrm{~B} 14,12, \mathrm{~A} 13,13, \mathrm{D} 12,0 \backslash \mathrm{H}, 8, \mathrm{~B} 15,1, \mathrm{~A} 14,2, \mathrm{D} 13,0 \backslash \mathrm{H}, 8, \mathrm{~B} 16,1, \mathrm{~A} 15,16, \mathrm{D} 1$ $4,0 \backslash \mathrm{H}, 8, \mathrm{~B} 17,1, \mathrm{~A} 16,16, \mathrm{D} 15,0 \backslash \mathrm{H}, 15, \mathrm{~B} 18,14, \mathrm{~A} 17,12, \mathrm{D} 16,0 \backslash \mathrm{H}, 15, \mathrm{~B} 19,14, \mathrm{~A} 18,19$ $, \mathrm{D} 17,0 \backslash \mathrm{H}, 15, \mathrm{~B} 20,14, \mathrm{~A} 19,19, \mathrm{D} 18,0 \backslash \backslash \mathrm{D} 5=-47.66210571 \backslash \mathrm{D} 6=124.2034009 \backslash \mathrm{D} 7=51$. $45345692 \backslash D 8=169.97852761 \backslash D 9=-69.03688301 \backslash D 10=79.59632628 \backslash D 11=-178.3012$ $6539 \backslash D 12=-1.10736436 \backslash D 13=-176.1569452 \backslash D 14=-118.70056952 \backslash D 15=118.684277$ $86 \backslash D 16=59.84254391 \backslash D 17=-120.7098879 \backslash D 18=119.71257159 \backslash B 1=1.75470101 \backslash B 2=$ $1.50144142 \backslash \mathrm{B} 3=1.7540458 \backslash \mathrm{B} 4=1.09580114 \backslash \mathrm{B} 5=1.101524 \backslash \mathrm{B} 6=1.09607085 \backslash \mathrm{B} 7=1.8$ $3909477 \backslash \mathrm{B} 8=1.85893744 \backslash \mathrm{B} 9=1.09152394 \backslash \mathrm{B} 10=1.09162639 \backslash \mathrm{B} 11=1.50894962 \backslash \mathrm{B} 12=$ $1.21538556 \backslash \mathrm{B} 13=1.34868612 \backslash \mathrm{B} 14=1.43745151 \backslash \mathrm{B} 15=1.09346144 \backslash \mathrm{B} 16=1.09284121$ $\backslash \mathrm{B} 17=1.09195971 \backslash \mathrm{B} 18=1.0931971 \backslash \mathrm{B} 19=1.09330407 \backslash \mathrm{B} 20=1.09019523 \backslash \mathrm{A} 1=122.104$ $41177 \backslash \mathrm{A} 2=118.7219121 \backslash \mathrm{A} 3=111.19302428 \backslash \mathrm{A} 4=111.34102025 \backslash \mathrm{A} 5=111.01263129 \backslash \mathrm{A}$ $6=102.5326864 \backslash A 7=103.45710004 \backslash A 8=108.16559651 \backslash A 9=105.69370446 \backslash A 10=110$. 
$77782324 \backslash \mathrm{A} 11=124.84467967 \backslash \mathrm{A} 12=111.49500109 \backslash \mathrm{A} 13=115.39415873 \backslash \mathrm{A} 14=106.25$ $208603 \backslash A 15=111.51685978 \backslash A 16=109.15493098 \backslash A 17=110.56758888 \backslash A 18=110.4339$ $1008 \backslash \mathrm{A} 19=105.51868249 \backslash \mathrm{D} 1=179.45105199 \backslash \mathrm{D} 2=-31.67767788 \backslash \mathrm{D} 3=120.39887003 \backslash$

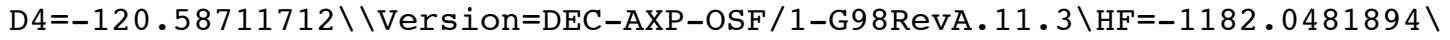
$\mathrm{S} 2=0.756239 \backslash \mathrm{S} 2-1=0 . \backslash \mathrm{S} 2 \mathrm{~A}=0.750029 \backslash \mathrm{RMSD}=0.000 \mathrm{e}+00 \backslash \mathrm{RMSF}=9.546 \mathrm{e}-05 \backslash \mathrm{Dipole}=$ $0.1388335,0.8127896,0.3170917 \backslash \mathrm{PG}=\mathrm{C} 01 \quad[\mathrm{X}(\mathrm{C} 6 \mathrm{H} 11 \mathrm{O} 2 \mathrm{~S} 2)] \backslash \backslash \mathrm{a}$

\section{$\mathrm{CH}_{3} \mathrm{SC} \cdot\left(\mathrm{CH}_{3}\right) \mathrm{S}-\mathrm{C}\left(\mathrm{CH}_{3}\right)_{2} \mathrm{CN}(6)$}

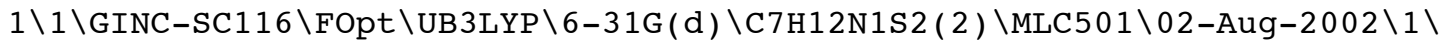
$\backslash \# N$ UB3LYP $/ 6-31 \mathrm{G}(\mathrm{D}) \mathrm{OPT}=(\mathrm{Z}-\mathrm{MATRIX}, \mathrm{MAXCYC}=100) \mathrm{SCF}=(\mathrm{MAXCYC}=100, \mathrm{QC}, \mathrm{TIGHT}$ ) FREQ MAXDISK=131072000\\RAFT_RAD( $\mathrm{Z}=\mathrm{Me} \mathrm{R}=\mathrm{Me} 2 \mathrm{CCN}) \mathrm{C} 1 \mathrm{~b} / / \mathrm{B} 3 \backslash \backslash 0,2 \backslash \mathrm{C} \backslash \mathrm{C}, 1, \mathrm{~B}$ $1 \backslash \mathrm{S}, 1, \mathrm{~B} 2,2, \mathrm{~A} 1 \backslash \mathrm{S}, 1, \mathrm{~B} 3,2, \mathrm{~A} 2,3, \mathrm{D} 1,0 \backslash \mathrm{C}, 3, \mathrm{~B} 4,1, \mathrm{~A} 3,2, \mathrm{D} 2,0 \backslash \mathrm{C}, 4, \mathrm{~B} 5,1, \mathrm{~A} 4,2, \mathrm{D} 3,0$ $\backslash \mathrm{C}, 6, \mathrm{~B} 6,4, \mathrm{~A} 5,1, \mathrm{D} 4,0 \backslash \mathrm{C}, 6, \mathrm{~B} 7,4, \mathrm{~A} 6,1, \mathrm{D} 5,0 \backslash \mathrm{C}, 6, \mathrm{~B} 8,4, \mathrm{~A} 7,1, \mathrm{D} 6,0 \backslash \mathrm{H}, 5, \mathrm{~B} 9,3, \mathrm{~A} 8$, $1, \mathrm{D} 7,0 \backslash \mathrm{H}, 5, \mathrm{~B} 10,3, \mathrm{~A} 9,10, \mathrm{D} 8,0 \backslash \mathrm{H}, 5, \mathrm{~B} 11,3, \mathrm{~A} 10,10, \mathrm{D} 9,0 \backslash \mathrm{H}, 2, \mathrm{~B} 12,1, \mathrm{~A} 11,3, \mathrm{D} 10$, $0 \backslash \mathrm{H}, 2, \mathrm{~B} 13,1, \mathrm{~A} 12,13, \mathrm{D} 11,0 \backslash \mathrm{H}, 2, \mathrm{~B} 14,1, \mathrm{~A} 13,13, \mathrm{D} 12,0 \backslash \mathrm{H}, 7, \mathrm{~B} 15,6, \mathrm{~A} 14,4, \mathrm{D} 13,0 \backslash$ $\mathrm{H}, 7, \mathrm{~B} 16,6, \mathrm{~A} 15,16, \mathrm{D} 14,0 \backslash \mathrm{H}, 7, \mathrm{~B} 17,6, \mathrm{~A} 16,16, \mathrm{D} 15,0 \backslash \mathrm{H}, 8, \mathrm{~B} 18,6, \mathrm{~A} 17,4, \mathrm{D} 16,0 \backslash \mathrm{H}$, $8, \mathrm{~B} 19,6, \mathrm{~A} 18,19, \mathrm{D} 17,0 \backslash \mathrm{H}, 8, \mathrm{~B} 20,6, \mathrm{~A} 19,19, \mathrm{D} 18,0 \backslash \mathrm{X}, 9,1 ., 6,90.4, \mathrm{D} 19,0 \backslash \mathrm{N}, 9, \mathrm{~B}$ $21,22, \mathrm{~A} 20,6,180 ., 0 \backslash \backslash \mathrm{D} 2=16.38295078 \backslash \mathrm{D} 3=77.64088756 \backslash \mathrm{D} 4=60.37483458 \backslash \mathrm{D} 5=-1$ $78.7383868 \backslash \mathrm{D} 6=-61.16988989 \backslash \mathrm{B} 1=1.50208945 \backslash \mathrm{B} 2=1.75657198 \backslash \mathrm{B} 3=1.74732399 \backslash \mathrm{B}$ $4=1.83034897 \backslash \mathrm{B} 5=1.91282884 \backslash \mathrm{B} 6=1.53539307 \backslash \mathrm{B} 7=1.5395273 \backslash \mathrm{B} 8=1.46607825 \backslash \mathrm{B} 9$ $=1.09303629 \backslash \mathrm{B} 10=1.092321 \backslash \mathrm{B} 11=1.09293827 \backslash \mathrm{B} 12=1.10007771 \backslash \mathrm{B} 13=1.09890754 \backslash$ $\mathrm{B} 14=1.09212294 \backslash \mathrm{B} 15=1.09292583 \backslash \mathrm{B} 16=1.09396533 \backslash \mathrm{B} 17=1.09672447 \backslash \mathrm{B} 18=1.0945$ $1538 \backslash \mathrm{B} 19=1.09522691 \backslash \mathrm{B} 20=1.09354597 \backslash \mathrm{B} 21=1.16247912 \backslash \mathrm{A} 1=122.50686981 \backslash \mathrm{A} 2=1$ $23.17398239 \backslash A 3=104.46655439 \backslash A 4=106.49858295 \backslash A 5=110.98235599 \backslash A 6=104.639$ $96652 \backslash A 7=108.61843008 \backslash A 8=105.70641764 \backslash A 9=111.77488663 \backslash A 10=111.17328243$ $\backslash A 11=112.07996327 \backslash A 12=111.90892444 \backslash A 13=110.10196343 \backslash A 14=111.36166171 \backslash A$ $15=109.52774174 \backslash A 16=110.12678 \backslash A 17=109.62280482 \backslash A 18=109.87509857 \backslash A 19=11$ $1.407113 \backslash \mathrm{A} 20=89.82090986 \backslash \mathrm{D} 1=-177.90491864 \backslash \mathrm{D} 7=180.96073931 \backslash \mathrm{D} 8=118.42528$ $654 \backslash D 9=-118.08928553 \backslash D 10=64.15148444 \backslash D 11=-120.40351119 \backslash D 12=119.4744608$ $\backslash D 13=-60.04758136 \backslash D 14=120.04550823 \backslash D 15=-120.27316252 \backslash D 16=-61.18845374 \backslash$ $\mathrm{D} 17=-119.2157694 \backslash \mathrm{D} 18=120.55449916 \backslash \mathrm{D} 19=166.9636757 \backslash \backslash$ Version=DEC-AXP-OSF $/ 1-G 98 R e v A .11 .3 \backslash \mathrm{HF}=-1125.0409306 \backslash \mathrm{S} 2=0.755753 \backslash \mathrm{S} 2-1=0 . \backslash \mathrm{S} 2 \mathrm{~A}=0.750025 \backslash \mathrm{RMSD}$ $=0.000 e+00 \backslash R M S F=9.570 e-05 \backslash D i p o l e=0.9525901,-0.1520827,-0.5410097 \backslash P G=C 0$ $1[\mathrm{X}(\mathrm{C} 7 \mathrm{H} 12 \mathrm{~N} 1 \mathrm{~S} 2)] \backslash \backslash \mathrm{Q}$

\section{$\cdot \mathrm{CH}_{3}(7)$}

$1 \backslash 1 \backslash G I N C-S C 116 \backslash F O p t \backslash U B 3 L Y P \backslash 6-31 G(d) \backslash C 1 H 3(2) \backslash R G B 501 \backslash 22-A u g-2002 \backslash 0 \backslash \backslash \# N B$ 3LYP 6-31G(D) SCF=TIGHT OPT=TIGHT FREQ MAXDISK=13107200\\methyl Opt\&Fr eq UB3-LYP $/ 6-31 \mathrm{G}(\mathrm{d}) \backslash \backslash 0,2 \backslash \mathrm{C}, 0.0 ., 0 . \backslash \mathrm{H}, 1.082752351,0 ., 0 . \backslash \mathrm{H},-0.541376175$ $5,-0.937691042,0 . \backslash \mathrm{H},-0.5413761755,0.937691042,0$. \\Version=DEC-AXP-OSF / $1-\mathrm{G} 98 \mathrm{RevA} .11 .3 \backslash \mathrm{HF}=-39.8382922 \backslash \mathrm{S} 2=0.753765 \backslash \mathrm{S} 2-1=0 . \backslash \mathrm{S} 2 \mathrm{~A}=0.750007 \backslash \mathrm{RMSD}=4$. $410 \mathrm{e}-09 \backslash \mathrm{RMSF}=1.426 \mathrm{e}-09 \backslash \mathrm{Dipole}=0 ., 0 ., 0 . \backslash \mathrm{PG}=\mathrm{D} 03 \mathrm{H} \quad[\mathrm{O}(\mathrm{C} 1), 3 \mathrm{C} 2(\mathrm{H} 1)] \backslash \backslash @$

\section{$\cdot \mathrm{CH}_{2} \mathrm{Ph}(8)$}

$1 \backslash 1 \backslash G I N C-S C 89 \backslash F O p t \backslash U B 3 L Y P \backslash 6-31 G(d) \backslash C 7 H 7(2) \backslash M L C 501 \backslash 18-J u 1-2002 \backslash 1 \backslash \backslash \#$ UB3 LYP $/ 6-31 \mathrm{G} * \mathrm{FOPT}=($ TIGHT $, \mathrm{MAXCYC}=100, \mathrm{Z}-\mathrm{MATRIX})$ MAXDISK $=131072000$ FREQ SCF $=($ TIGHT, MAXCYC $=100) \backslash \backslash \mathrm{bz}$ rad-c2v-6dub3 (guess from dave) $\backslash \backslash 0,2 \backslash \mathrm{C} \backslash \mathrm{C}, 1, \mathrm{~B} 1 \backslash$ $\mathrm{C}, 2, \mathrm{~B} 2,1, \mathrm{~A} 1 \backslash \mathrm{C}, 2, \mathrm{~B} 2,1, \mathrm{~A} 1,3,-180 ., 0 \backslash \mathrm{C}, 3, \mathrm{~B} 3,2, \mathrm{~A} 2,1,-180$. $0 \backslash \mathrm{C}, 4, \mathrm{~B} 3,2, \mathrm{~A} 2,1$, $180 ., 0 \backslash \mathrm{H}, 1, \mathrm{~B} 4,2, \mathrm{~A} 3,3,0 ., 0 \backslash \mathrm{H}, 1, \mathrm{~B} 4,2, \mathrm{~A} 3,7,-180.0 \backslash \mathrm{H}, 3, \mathrm{~B} 5,2, \mathrm{~A} 4,5,180 ., 0 \backslash \mathrm{H}$ $, 4, \mathrm{~B} 5,2, \mathrm{~A} 4,6,-180 ., 0 \backslash \mathrm{H}, 5, \mathrm{~B} 6,3, \mathrm{~A} 5,2,180 ., 0 \backslash \mathrm{H}, 6, \mathrm{~B} 6,4, \mathrm{~A} 5,2,-180 ., 0 \backslash \mathrm{X}, 2,1$. $, 1,90 ., 3,90 ., 0 \backslash \mathrm{C}, 2, \mathrm{~B} 7,13,90 ., 1,180 ., 0 \backslash \mathrm{X}, 14,1 ., 2,90.13,0 ., 0 \backslash \mathrm{H}, 14, \mathrm{~B} 8,15$ $, 90 ., 2,180 ., 0 \backslash \backslash \mathrm{B} 1=1.40658309 \backslash \mathrm{B} 2=1.4269787 \backslash \mathrm{B} 3=1.38585898 \backslash \mathrm{B} 4=1.08519274 \backslash$ $\mathrm{B} 5=1.08733663 \backslash \mathrm{B} 6=1.08707607 \backslash \mathrm{B} 7=2.83514342 \backslash \mathrm{B} 8=1.08628629 \backslash \mathrm{A} 1=121.3932742$ $5 \backslash A 2=121.13892253 \backslash A 3=121.22638869 \backslash A 4=118.71171974 \backslash A 5=119.70158608 \backslash \backslash$ Ver

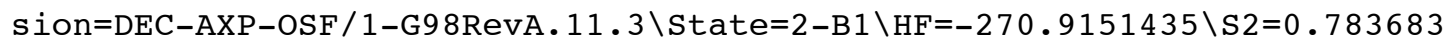
$\backslash S 2-1=0 . \backslash S 2 A=0.750769 \backslash R M S D=5.840 e-09 \backslash R M S F=5.706 e-07 \backslash D i p o l e=0,0 ., 0.052$ $5474 \backslash \mathrm{PG}=\mathrm{C} 02 \mathrm{~V} \quad[\mathrm{C} 2(\mathrm{H} 1 \mathrm{C} 1 \mathrm{C} 1 \mathrm{C} 1), \mathrm{SGV}(\mathrm{C} 4 \mathrm{H} 6)] \backslash \backslash @$ 


\section{$\cdot \mathrm{C}\left(\mathrm{CH}_{3}\right) \mathrm{CN}(9)$}

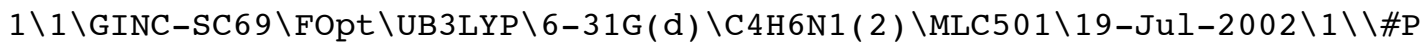
UB3LYP /6-31G* FOPT $=(Z-M A T R I X, T I G H T, M A X C Y C=100)$ FREQ MAXDISK=131072000 $\mathrm{SCF}=(\mathrm{TIGHT}, \mathrm{MAXCYC}=100) \backslash \backslash \mathrm{me} 2 \mathrm{cCn}-\mathrm{C} 2 \mathrm{vb}-6 \mathrm{dub} 3 \backslash \backslash 0,2 \backslash \mathrm{C} \backslash \mathrm{C}, 1, \mathrm{~b} 1 \backslash \mathrm{X}, 2,1 ., 1,90 . \backslash \mathrm{N}$ $, 2, \mathrm{~b} 2,3,90 \ldots, 1,180 \ldots, 0 \backslash \mathrm{C}, 1, \mathrm{~b} 3,2, \mathrm{a} 2,3,0 ., 0 \backslash \mathrm{C}, 1, \mathrm{~b} 3,2, \mathrm{a} 2,3,180 ., 0 \backslash \mathrm{H}, 5, \mathrm{~b} 4,1$, $\mathrm{a} 3,2,0 ., 0 \backslash \mathrm{H}, 6, \mathrm{~b} 4,1, \mathrm{a} 3,2,0 ., 0 \backslash \mathrm{H}, 5, \mathrm{~b} 5,1, \mathrm{a} 4,7, \mathrm{~d} 1,0 \backslash \mathrm{H}, 5, \mathrm{~b} 5,1, \mathrm{a} 4,7,-\mathrm{d} 1,0 \backslash \mathrm{H}$, $6, \mathrm{~b} 5,1, \mathrm{a} 4,8, \mathrm{~d} 1,0 \backslash \mathrm{H}, 6, \mathrm{~b} 5,1, \mathrm{a} 4,8,-\mathrm{d} 1,0 \backslash \backslash \mathrm{b} 1=1.39173287 \backslash \mathrm{b} 2=1.17666605 \backslash \mathrm{b} 3=1$ $.50156142 \backslash \mathrm{b} 4=1.09243341 \backslash \mathrm{b} 5=1.09976071 \backslash \mathrm{a} 2=120.24203494 \backslash \mathrm{a} 3=112.14994686 \backslash$ $\mathrm{a} 4=110.62200038 \backslash \mathrm{d} 1=121.09506187 \backslash \backslash$ Version=DEC-AXP-OSF $/ 1-G 98 \mathrm{ReVA} .11 .3 \backslash \mathrm{St}$ ate $=2-B 1 \backslash \mathrm{HF}=-210.7373225 \backslash \mathrm{S} 2=0.765669 \backslash \mathrm{S} 2-1=0 . \backslash \mathrm{S} 2 \mathrm{~A}=0.750113 \backslash \mathrm{RMSD}=8.850 \mathrm{e}-$ $09 \backslash \mathrm{RMSF}=1.667 \mathrm{e}-06 \backslash \mathrm{Dipole}=0 ., 0 .,-1.623572 \backslash \mathrm{PG}=\mathrm{C} 02 \mathrm{~V} \quad[\mathrm{C} 2(\mathrm{C} 1 \mathrm{C} 1 \mathrm{~N} 1), \mathrm{SGV}(\mathrm{C} 2 \mathrm{H} 2)$ $\mathrm{X}(\mathrm{H} 4)] \backslash \backslash \mathrm{Q}$

\section{$\cdot \mathrm{CH}_{2} \mathrm{COOCH}_{3}(10)$}

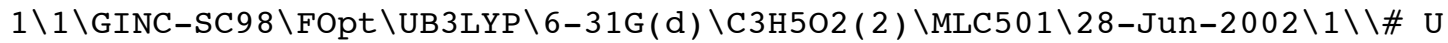
B3LYP /6-31G* FOPT $=(Z-M A T R I X, M A X C Y C=100$, TIGHT $)$ MAXDISK=65536000 FREQ SC $\mathrm{F}=(\mathrm{TIGHT}, \mathrm{MAXCYC}=100) \backslash \backslash \mathrm{ch} 2 \mathrm{cooch} 3 \mathrm{rad}(\mathrm{MA}) \mathrm{Cs} \backslash \backslash 0,2 \backslash \mathrm{C} \backslash \mathrm{C}, 1, \mathrm{~B} 1 \backslash 0,2, \mathrm{~B} 2,1, \mathrm{~A} 1 \backslash$ $\mathrm{O}, 2, \mathrm{~B} 3,1, \mathrm{~A} 2,3,-180 ., 0 \backslash \mathrm{C}, 4, \mathrm{~B} 4,2, \mathrm{~A} 3,1,180.0 \backslash \mathrm{H}, 5, \mathrm{~B} 5,4, \mathrm{~A} 4,2,-180 ., 0 \backslash \mathrm{H}, 1, \mathrm{~B}$ $6,2, \mathrm{~A} 5,3,180 ., 0 \backslash \mathrm{H}, 1, \mathrm{~B} 7,2, \mathrm{~A} 6,7,-180 ., 0 \backslash \mathrm{H}, 5, \mathrm{~B} 8,4, \mathrm{~A} 7,6, \mathrm{D} 1,0 \backslash \mathrm{H}, 5, \mathrm{~B} 8,4, \mathrm{~A} 7,6$ $,-\mathrm{D} 1,0 \backslash \backslash \mathrm{B} 1=1.44732926 \backslash \mathrm{B} 2=1.22312018 \backslash \mathrm{B} 3=1.36100596 \backslash \mathrm{B} 4=1.43336725 \backslash \mathrm{B} 5=1.0$ $9058408 \backslash B 6=1.0833186 \backslash B 7=1.0824072 \backslash B 8=1.09394961 \backslash A 1=124.74743883 \backslash A 2=111$ $.87082202 \backslash A 3=114.93235024 \backslash A 4=105.62119929 \backslash A 5=121.24789916 \backslash A 6=118.31516$ $036 \backslash A 7=110.72623732 \backslash D 1=119.68853085 \backslash \backslash$ Version=DEC-AXP-OSF / 1-G98RevA. 11 . $3 \backslash$ State $=2-\mathrm{A} " \backslash \mathrm{HF}=-267.7237418 \backslash \mathrm{S} 2=0.757594 \backslash \mathrm{S} 2-1=0 . \backslash \mathrm{S} 2 \mathrm{~A}=0.750034 \backslash \mathrm{RMSD}=4.6$ $63 e-09 \backslash \mathrm{RMSF}=3.942 \mathrm{e}-06 \backslash \mathrm{Dipole}=-0.6413843,0,,-0.2480326 \backslash \mathrm{PG}=\mathrm{CS} \quad[\mathrm{SG}(\mathrm{C} 3 \mathrm{H} 3 \mathrm{O} 2$ ), $\mathrm{X}(\mathrm{H} 2) \mathrm{]} \backslash \backslash \mathrm{Q}$

\section{$\mathrm{S}=\mathrm{C}\left(\mathrm{CH}_{3}\right) \mathrm{SCH}_{3}(11)$}

$1 \backslash 1 \backslash G I N C-S C 100 \backslash F O p t \backslash R B 3 L Y P \backslash 6-31 G(d) \backslash C 3 H 6 S 2 \backslash M L C 501 \backslash 17-J u 1-2002 \backslash 1 \backslash \backslash \# P R B$ $3 \mathrm{LYP} / 6-31 \mathrm{G} * \mathrm{FOPT}=(\mathrm{MAXCYC}=150, \mathrm{Z}-\mathrm{MATRIX}) \quad \mathrm{MAXDISK}=131072000 \mathrm{FREQ} \quad \mathrm{SCF}=\mathrm{TIGH}$ $\mathrm{T}$ GEOM=CHECK GUESS=READ $\backslash \backslash \mathrm{S}=\mathrm{C}(\mathrm{Me})-\mathrm{S}-\mathrm{Me} \mathrm{C} 1 \backslash \backslash 0,1 \backslash \mathrm{S} \backslash \mathrm{C}, 1, \mathrm{~b} 1 \backslash \mathrm{C}, 2, \mathrm{~b} 2,1, \mathrm{a} 1 \backslash \mathrm{S}, 2$ , b3 , $1, \mathrm{a} 2,3$, dh $3,0 \backslash \mathrm{C}, 4, \mathrm{~b} 4,2, \mathrm{a} 3,1, \mathrm{dh} 4,0 \backslash \mathrm{H}, 5, \mathrm{~b} 5,4, \mathrm{a} 4,2$, dh $5,0 \backslash \mathrm{H}, 5, \mathrm{~b} 6,4, \mathrm{a} 5,2$ , dh $1,0 \backslash \mathrm{H}, 5, \mathrm{~b} 6 \mathrm{a}, 4, \mathrm{a} 5 \mathrm{a}, 2,-\mathrm{dh} 1 \mathrm{a}, 0 \backslash \mathrm{H}, 3, \mathrm{~b} 7,2, \mathrm{a} 6,1, \mathrm{dh} 6,0 \backslash \mathrm{H}, 3, \mathrm{~b} 8,2, \mathrm{a} 7,1, \mathrm{dh} 2,0$ $\backslash \mathrm{H}, 3, \mathrm{~b} 8 \mathrm{a}, 2, \mathrm{a} 7 \mathrm{a}, 1,-\mathrm{dh} 2 \mathrm{a}, 0 \backslash \backslash \mathrm{b} 1=1.64599636 \backslash \mathrm{b} 2=1.5148461 \backslash \mathrm{b} 3=1.75511783 \backslash \mathrm{b} 4=$ $1.81794969 \backslash \mathrm{b} 5=1.09307671 \backslash \mathrm{b} 6=1.0919738 \backslash \mathrm{b} 6 \mathrm{a}=1.09197036 \backslash \mathrm{b} 7=1.09324611 \backslash \mathrm{b} 8=$ $1.09537804 \backslash \mathrm{b} 8 \mathrm{a}=1.0962657 \backslash \mathrm{a} 1=122.84648556 \backslash \mathrm{a} 2=125.75342086 \backslash \mathrm{a} 3=103.776055$ $3 \backslash a 4=106.19073532 \backslash a 5=110.33849971 \backslash a 5 a=110.27255399 \backslash a 6=109.69980988 \backslash a 7=$ $112.81878244 \backslash a 7 a=109.03573022 \backslash d h 1=60.70823437 \backslash d h 1 a=60.13720525 \backslash d h 2=167$ $.56947484 \backslash \mathrm{dh} 2 \mathrm{a}=72.03339216 \backslash \mathrm{dh} 3=181.23401229 \backslash \mathrm{dh} 4=0.90237034 \backslash \mathrm{dh} 5=180.305$ $77272 \backslash \mathrm{dh} 6=45.4338183 \backslash \backslash$ Version=DEC-AXP-OSF $/ 1-G 98 \mathrm{RevA} .11 .3 \backslash \mathrm{HF}=-914.30737$ $69 \backslash \mathrm{RMSD}=3.465 \mathrm{e}-10 \backslash \mathrm{RMSF}=1.676 \mathrm{e}-06 \backslash \mathrm{Dipole}=-0.3544958,-0.0417535,0.694415$ $9 \backslash \mathrm{PG}=\mathrm{C} 01 \quad[\mathrm{X}(\mathrm{C} 3 \mathrm{H} 6 \mathrm{~S} 2)] \backslash \backslash \mathrm{Q}$

\section{$\mathrm{S}=\mathrm{C}(\mathrm{Ph}) \mathrm{SCH}_{3}(12)$}

$1 \backslash 1 \backslash G I N C-S C 116 \backslash F O p t \backslash R B 3 L Y P \backslash 6-31 G(d) \backslash C 8 H 8 S 2 \backslash M L C 501 \backslash 25-J u 1-2002 \backslash 1 \backslash \backslash \# P$ B3 LYP /6-31G* OPT $=(Z-M A T R I X, M A X C Y C=100)$ FREQ MAXDISK=131072000\\RAFT agen t $\mathrm{S}=\mathrm{C}(\mathrm{Ph})-\mathrm{S}-\mathrm{CH} 3 \quad(\mathrm{Z}=\mathrm{ph}, \mathrm{R}=\mathrm{me}) \backslash \backslash 0,1 \backslash \mathrm{C} \backslash \mathrm{S}, 1, \mathrm{~B} 1 \backslash \mathrm{S}, 1, \mathrm{~B} 2,2, \mathrm{~A} 1 \backslash \mathrm{C}, 3, \mathrm{~B} 3,1, \mathrm{~A} 2,2, \mathrm{D} 1$ $, 0 \backslash \mathrm{H}, 4, \mathrm{~B} 4,3, \mathrm{~A} 3,1, \mathrm{D} 2,0 \backslash \mathrm{H}, 4, \mathrm{~B} 5,3, \mathrm{~A} 4,5, \mathrm{D} 3,0 \backslash \mathrm{H}, 4, \mathrm{~B} 6,3, \mathrm{~A} 5,5, \mathrm{D} 4,0 \backslash \mathrm{C}, 1, \mathrm{~B} 7,2, \mathrm{~A}$ $6,3, \mathrm{D} 5,0 \backslash \mathrm{C}, 8, \mathrm{~B} 8,1, \mathrm{~A} 7,2, \mathrm{D} 6,0 \backslash \mathrm{C}, 9, \mathrm{~B} 9,8, \mathrm{~A} 8,1, \mathrm{D} 7,0 \backslash \mathrm{C}, 10, \mathrm{~B} 10,9, \mathrm{~A} 9,8, \mathrm{D} 8,0 \backslash \mathrm{C}$, $11, \mathrm{~B} 11,10, \mathrm{~A} 10,9, \mathrm{D} 9,0 \backslash \mathrm{C}, 12, \mathrm{~B} 12,11, \mathrm{~A} 11,10, \mathrm{D} 10,0 \backslash \mathrm{H}, 13, \mathrm{~B} 13,12, \mathrm{~A} 12,11, \mathrm{D} 11,0$ $\backslash \mathrm{H}, 12, \mathrm{~B} 14,11, \mathrm{~A} 13,13, \mathrm{D} 12,0 \backslash \mathrm{H}, 11, \mathrm{~B} 15,10, \mathrm{~A} 14,12, \mathrm{D} 13,0 \backslash \mathrm{H}, 10, \mathrm{~B} 16,9, \mathrm{~A} 15,11, \mathrm{D}$ $14,0 \backslash \mathrm{H}, 9, \mathrm{~B} 17,8, \mathrm{~A} 16,10, \mathrm{D} 15,0 \backslash \backslash \mathrm{B} 1=1.65390665 \backslash \mathrm{B} 2=1.76783874 \backslash \mathrm{B} 3=1.81735533$ $\backslash \mathrm{B} 4=1.09340644 \backslash \mathrm{B} 5=1.09203382 \backslash \mathrm{B} 6=1.09167768 \backslash \mathrm{B} 7=1.48638437 \backslash \mathrm{B} 8=1.40791986$ $\backslash \mathrm{B} 9=1.3934782 \backslash \mathrm{B} 10=1.3954032 \backslash \mathrm{B} 11=1.39756856 \backslash \mathrm{B} 12=1.39093249 \backslash \mathrm{B} 13=1.084558$ $18 \backslash \mathrm{B} 14=1.08646979 \backslash \mathrm{B} 15=1.08663117 \backslash \mathrm{B} 16=1.08643383 \backslash \mathrm{B} 17=1.08519217 \backslash \mathrm{A} 1=123$. $8831348 \backslash A 2=103.44785578 \backslash A 3=105.74311227 \backslash A 4=110.56367906 \backslash A 5=110.5050450$ 
$1 \backslash \mathrm{A} 6=123.43517028 \backslash \mathrm{A} 7=121.7784707 \backslash \mathrm{A} 8=120.59384832 \backslash \mathrm{A} 9=120.24074703 \backslash \mathrm{A} 10=1$ $19.68786961 \backslash \mathrm{A} 11=120.27839448 \backslash \mathrm{A} 12=120.55619114 \backslash \mathrm{A} 13=120.1204252 \backslash \mathrm{A} 14=120$. $14506072 \backslash \mathrm{A} 15=119.5622211 \backslash \mathrm{A} 16=119.91357919 \backslash \mathrm{D} 1=5.07451867 \backslash \mathrm{D} 2=178.7658797$ $5 \backslash D 3=-119.43094734 \backslash D 4=119.41965567 \backslash D 5=181.27901545 \backslash D 6=211.48944504 \backslash D 7=$ $179.74862327 \backslash \mathrm{D} 8=0.5087189 \backslash \mathrm{D} 9=-0.47334322 \backslash \mathrm{D} 10=-0.1123593 \backslash \mathrm{D} 11=180.118421$ $67 \backslash D 12=179.95923577 \backslash D 13=180.24754199 \backslash D 14=180.47358662 \backslash D 15=182.12059766$ $\backslash \backslash$ Version=DEC-AXP-OSF /1-G98RevA.11.3\HF=-1106.0452545 $\backslash \mathrm{RMSD}=3.885 \mathrm{e}-09 \backslash \mathrm{R}$ $\mathrm{MSF}=1.915 \mathrm{e}-05 \backslash \mathrm{Dipole}=0.0516049,-0.0290079,-0.8060901 \backslash \mathrm{PG}=\mathrm{C} 01 \quad[\mathrm{X}(\mathrm{C} 8 \mathrm{H} 8 \mathrm{~S} 2)$ ] $\backslash @$

\section{$\mathrm{S}=\mathrm{C}\left(\mathrm{CH}_{2} \mathrm{Ph}\right) \mathrm{SCH}_{3}(13)$}

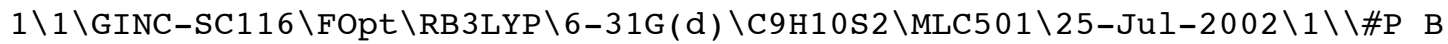
3LYP $/ 6-31 \mathrm{G}$ * OPT $=(\mathrm{Z}-\mathrm{MATRIX}, \mathrm{MAXCYC}=100)$ FREQ MAXDISK $=131072000 \backslash \backslash \mathrm{RAFT}$ Age nt $\mathrm{S}=\mathrm{C}(\mathrm{MePH})-\mathrm{SMe}(\mathrm{Z}=\mathrm{MePh}, \mathrm{R}=\mathrm{Me}) \mathrm{C} 1 \backslash \backslash 0,1 \backslash \mathrm{C} \backslash \mathrm{S}, 1, \mathrm{~B} 1 \backslash \mathrm{C}, 1, \mathrm{~B} 2,2, \mathrm{~A} 1 \backslash \mathrm{H}, 3, \mathrm{~B} 3,1$, $\mathrm{A} 2,2, \mathrm{D} 1,0 \backslash \mathrm{H}, 3, \mathrm{~B} 4,1, \mathrm{~A} 3,4, \mathrm{D} 2,0 \backslash \mathrm{C}, 3, \mathrm{~B} 5,1, \mathrm{~A} 4,4, \mathrm{D} 3,0 \backslash \mathrm{C}, 6, \mathrm{~B} 6,3, \mathrm{~A} 5,1, \mathrm{D} 4,0 \backslash \mathrm{C}, 7$ , $\mathrm{B} 7,6, \mathrm{~A} 6,3, \mathrm{D} 5,0 \backslash \mathrm{C}, 8, \mathrm{~B} 8,7, \mathrm{~A} 7,6, \mathrm{D} 6,0 \backslash \mathrm{C}, 9, \mathrm{~B} 9,8, \mathrm{~A} 8,7, \mathrm{D} 7,0 \backslash \mathrm{C}, 10, \mathrm{~B} 10,9, \mathrm{~A} 9,8$, $\mathrm{D} 8,0 \backslash \mathrm{H}, 11, \mathrm{~B} 11,10, \mathrm{~A} 10,9, \mathrm{D} 9,0 \backslash \mathrm{H}, 10, \mathrm{~B} 12,9, \mathrm{~A} 11,11, \mathrm{D} 10,0 \backslash \mathrm{H}, 9, \mathrm{~B} 13,8, \mathrm{~A} 12,10, \mathrm{D}$ $11,0 \backslash \mathrm{H}, 8, \mathrm{~B} 14,7, \mathrm{~A} 13,9, \mathrm{D} 12,0 \backslash \mathrm{H}, 7, \mathrm{~B} 15,6, \mathrm{~A} 14,8, \mathrm{D} 13,0 \backslash \mathrm{S}, 1, \mathrm{~B} 16,2, \mathrm{~A} 15,3, \mathrm{D} 14,0$ $\backslash \mathrm{C}, 17, \mathrm{~B} 17,1, \mathrm{~A} 16,2, \mathrm{D} 15,0 \backslash \mathrm{H}, 18, \mathrm{~B} 18,16, \mathrm{~A} 17,1, \mathrm{D} 16,0 \backslash \mathrm{H}, 18, \mathrm{~B} 19,16, \mathrm{~A} 18,1, \mathrm{D} 17$, $0 \backslash \mathrm{H}, 18, \mathrm{~B} 20,16, \mathrm{~A} 19,1, \mathrm{D} 18,0 \backslash \backslash \mathrm{B} 1=1.64564466 \backslash \mathrm{B} 2=1.52863415 \backslash \mathrm{B} 3=1.09674628 \backslash \mathrm{B}$ $4=1.09353236 \backslash B 5=1.52343747 \backslash B 6=1.39968158 \backslash B 7=1.39594042 \backslash B 8=1.39529816 \backslash B$ $9=1.39667801 \backslash \mathrm{B} 10=1.3946779 \backslash \mathrm{B} 11=1.08659727 \backslash \mathrm{B} 12=1.08682646 \backslash \mathrm{B} 13=1.0867289$ $4 \backslash \mathrm{B} 14=1.08683319 \backslash \mathrm{B} 15=1.08756909 \backslash \mathrm{B} 16=1.7547672 \backslash \mathrm{B} 17=1.81824902 \backslash \mathrm{B} 18=1.093$ $09254 \backslash B 19=1.09194447 \backslash B 20=1.0919036 \backslash A 1=123.05916516 \backslash A 2=109.80486391 \backslash A 3=$ $107.35736989 \backslash A 4=112.35357201 \backslash A 5=120.55086294 \backslash A 6=120.654591 \backslash A 7=120.0667$ $1159 \backslash A 8=119.64159031 \backslash A 9=120.22881755 \backslash A 10=120.05599167 \backslash A 11=120.09413459$ $\backslash A 12=120.18319366 \backslash A 13=119.73589867 \backslash A 14=119.61324586 \backslash A 15=125.66992548 \backslash A$ $16=103.79835693 \backslash A 17=105.33482935 \backslash A 18=93.82431614 \backslash A 19=126.14621 \backslash D 1=150$. $7138342 \backslash D 2=-116.89084223 \backslash D 3=121.89888903 \backslash D 4=-100.14574145 \backslash D 5=179.75805$ $35 \backslash D 6=-0.07990219 \backslash D 7=0.06511684 \backslash D 8=-0.01890592 \backslash D 9=180.33270637 \backslash D 10=179$ $.82110496 \backslash \mathrm{D} 11=-180.19616308 \backslash \mathrm{D} 12=179.8418732 \backslash \mathrm{D} 13=179.81914239 \backslash \mathrm{D} 14=-178$. $68759297 \backslash \mathrm{D} 15=2.34439814 \backslash \mathrm{D} 16=197.73877987 \backslash \mathrm{D} 17=85.44790483 \backslash \mathrm{D} 18=-31.96744$ 553 \Version=DEC-AXP-OSF / 1-G98RevA. 11.3 $\backslash \mathrm{HF}=-1145.3555037 \backslash \mathrm{RMSD}=4.812 \mathrm{e}-0$ $9 \backslash \mathrm{RMSF}=1.589 e-05 \backslash \mathrm{Dipole}=-0.341854,0.1539887,-0.6355984 \backslash \mathrm{PG}=\mathrm{C} 01] \mathrm{X}(\mathrm{C} 9 \mathrm{H} 10$ $\mathrm{S} 2)] \backslash \backslash$ Q

\section{$\mathrm{CH}_{3} \mathrm{SC} \cdot\left(\mathrm{CH}_{2} \mathrm{Ph}\right) \mathrm{S}-\mathrm{CH}_{2} \mathrm{Ph}(14)$}

$1 \backslash 1 \backslash G I N C-S C 47 \backslash F O p t \backslash U B 3 L Y P \backslash 6-31 G(d) \backslash C 16 H 17 S 2(2) \backslash M L C 501 \backslash 29-N o v-2002 \backslash 0 \backslash \backslash \#$ $\mathrm{N}$ B3LYP/6-31G(D) OPT $=($ TIGHT, $\mathrm{MAXCYC}=100) \quad \mathrm{SCF}=(\mathrm{QC}, \mathrm{TIGHT}, \mathrm{MAXCYC}=100)$ MAXD ISK=131072000 FREQ GUESS=READ GEOM=CHECK \\RRAD(Z=Bz R=Bz) c1bx1 s2=.77 $\backslash \backslash 0,2 \backslash \mathrm{C},-1.4614717747,1.4086931623,0.6848456579 \backslash \mathrm{S},-2.0993383706,2.6758$ $683047,-0.3570429371 \backslash \mathrm{S}, 0.2426341659,1.1643700406,0.9973054226 \backslash \mathrm{C},-2.487$ $2031109,0.4013895222,1.1683663781 \backslash \mathrm{C},-2.39861676,-0.9493788684,0.470116$ $8438 \backslash \mathrm{H},-3.4938332437,0.8201491417,1.037236164 \backslash \mathrm{H},-2.347500676,0.2545186$ $846,2.2475247709 \backslash \mathrm{C},-0.7422527595,3.8879466355,-0.4945029918 \backslash \mathrm{C}, 0.879550$ $1234,0.2873118183,-0.5573407052 \backslash \mathrm{C}, 2.3166736297,-0.094444675,-0.3563102$ $655 \backslash \mathrm{H}, 0.2436160083,-0.5859298621,-0.7114423114 \backslash \mathrm{H}, 0.755363173,0.9739204$ $757,-1.3990074679 \backslash \mathrm{H},-1.1473140531,4.7220977689,-1.0737257985 \backslash \mathrm{H},-0.4387$ $731907,4.2380497168,0.4941021144 \backslash \mathrm{H}, 0.1205613235,3.4682449182,-1.014720$ $1497 \backslash \mathrm{C}, 3.3509401549,0.7931412544,-0.6859386387 \backslash \mathrm{C}, 4.685113191,0.4402724$ $182,-0.4842761492 \backslash \mathrm{C}, 5.0052918379,-0.8070223139,0.0553286185 \backslash \mathrm{C}, 3.983471$ $9902,-1.6975229568,0.3923525525 \backslash \mathrm{C}, 2.6503787509,-1.3421075003,0.1908451$ $672 \backslash \mathrm{H}, 1.8552622996,-2.0358671613,0.4534328817 \backslash \mathrm{H}, 4.2253900578,-2.671018$ $0389,0.8108179316 \backslash \mathrm{H}, 6.0445514041,-1.0842405334,0.2105355045 \backslash \mathrm{H}, 5.474667$ $0885,1.1381039349,-0.7506093318 \backslash \mathrm{H}, 3.1063314933,1.7653958577,-1.1079215$ $117 \backslash \mathrm{C},-1.8103559569,-2.048548613,1.1079998748 \backslash \mathrm{C},-1.7010227203,-3.27981$ $89345,0.4566411093 \backslash \mathrm{C},-2.1826605875,-3.4288896931,-0.8445460339 \backslash \mathrm{C},-2.77$ $56543255,-2.3400638306,-1.488951393 \backslash \mathrm{C},-2.8808651348,-1.1117347639,-0.8$ 
$367252567 \backslash \mathrm{H},-3.3397562395,-0.267515043,-1.3466083656 \backslash \mathrm{H},-3.1586150331$,$2.4484389855,-2.5004940611 \backslash \mathrm{H},-2.1012114503,-4.3862937257,-1.3522571907$ $\backslash \mathrm{H},-1.244808637,-4.1228848337,0.9693940127 \backslash \mathrm{H},-1.4345623372,-1.93744180$ $77,2.1221144314 \backslash \backslash$ Version=DEC-AXP-OSF / 1-G98RevA. $11.3 \backslash \mathrm{HF}=-1416.2768267 \backslash \mathrm{S}$ $2=0.755837 \backslash \mathrm{S} 2-1=0 . \backslash \mathrm{S} 2 \mathrm{~A}=0.750027 \backslash \mathrm{RMSD}=0.000 e+00 \backslash \mathrm{RMSF}=3.566 \mathrm{e}-06 \backslash \mathrm{Dipole}=0$ $.2523139,-0.0076248,-0.4348361 \backslash \mathrm{PG}=\mathrm{C} 01 \quad[\mathrm{X}(\mathrm{C} 16 \mathrm{H} 17 \mathrm{~S} 2)] \backslash \backslash \mathrm{a}$

\section{$\mathrm{CH}_{3} \mathrm{SC} \cdot(\mathrm{Ph}) \mathrm{S}-\mathrm{CH}_{2} \mathrm{Ph}(15)$}

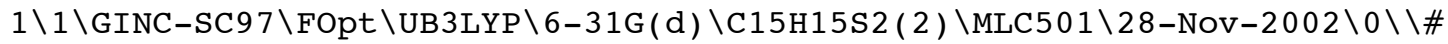
$\mathrm{N}$ B3LYP/6-31G(D) OPT $=($ TIGHT, $\operatorname{MAXCYC}=100) \quad \mathrm{SCF}=(\mathrm{QC}, \mathrm{TIGHT}, \mathrm{MAXCYC}=100)$ MAXD $I S K=131072000$ FREQ $\backslash \backslash \operatorname{RRAD}(\mathrm{Z}=\mathrm{Ph} \mathrm{R}=\mathrm{Bz}) \mathrm{c} 1 \mathrm{cx} \quad \mathrm{s} 2=1.39 \backslash \backslash 0,2 \backslash \mathrm{C},-0.0277767217$, $-1.2203997432,0.5999659775 \backslash S,-0.0489339369,-1.1725510745,2.3706549281 \backslash$ $\mathrm{S}, 1.5174615623,-1.2123976173,-0.2517610955 \backslash \mathrm{C},-1.2757135254,-1.23635292$ $82,-0.1279986748 \backslash \mathrm{C}, 1.1932292566,-2.4302587846,2.8608967633 \backslash \mathrm{C}, 2.3656778$ $049,0.3407983455,0.3592944891 \backslash \mathrm{C}, 1.7974110213,1.6132335008,-0.203441073$ $4 \backslash \mathrm{H}, 2.3253234754,0.3299147564,1.4513370916 \backslash \mathrm{H}, 3.4053249361,0.1913857073$ , $0.0509755449 \backslash \mathrm{C}, 0.8500636,2.3505487406,0.5197155057 \backslash \mathrm{C}, 2.193142809,2.07$ $918905,-1.4657520982 \backslash \mathrm{C}, 0.3098184235,3.5246527245,-0.0071990753 \backslash \mathrm{C}, 1.655$ $899676,3.2523590906,-1.9926199251 \backslash \mathrm{C}, 0.7105213426,3.978974466,-1.264266$ $8502 \backslash \mathrm{H}, 0.5379640569,2.0012333502,1.5006094463 \backslash \mathrm{H}, 2.9270105505,1.5157890$ $807,-2.0381346404 \backslash \mathrm{H},-0.4232432988,4.085232446,0.5668888337 \backslash \mathrm{H}, 1.9770908$ $217,3.601961753,-2.9703768011 \backslash \mathrm{H}, 0.2918446249,4.8943967924,-1.673737885$ $1 \backslash \mathrm{C},-2.4940634039,-1.631793379,0.4908609075 \backslash \mathrm{C},-1.3392913215,-0.8573744$ $494,-1.4974927996 \backslash \mathrm{C},-3.6891419087,-1.643844879,-0.2156252782 \backslash \mathrm{C},-2.5401$ $242477,-0.8764732527,-2.1932837917 \backslash \mathrm{C},-3.7263902812,-1.2684046796,-1.56$ $28470183 \backslash \mathrm{H},-2.4805249598,-1.9437411163,1.5291937117 \backslash \mathrm{H},-0.43278549,-0.5$ $313131429,-1.9942815471 \backslash \mathrm{H},-4.6008338385,-1.9578281582,0.2863612257 \backslash \mathrm{H},-$ $2.5545015779,-0.573491869,-3.2371422 \backslash \mathrm{H},-4.6637044525,-1.2807923567,-2$. $1120465999 \backslash \mathrm{H}, 1.2468675007,-2.3890673441,3.9522192321 \backslash \mathrm{H}, 2.1737451597,-2$ $.2066557695,2.4373239658 \backslash \mathrm{H}, 0.8744053437,-3.4269679938,2.5472669489 \backslash \backslash \mathrm{Ve}$ rsion=DEC-AXP-OSF $/ 1-G 98 R e v A .11 .3 \backslash \mathrm{HF}=-1376.9749829 \backslash \mathrm{S} 2=0.771784 \backslash \mathrm{S} 2-1=0 . \backslash$ $\mathrm{S} 2 \mathrm{~A}=0.750355 \backslash \mathrm{RMSD}=0.000 \mathrm{e}+00 \backslash \mathrm{RMSF}=2.549 \mathrm{e}-07 \backslash \mathrm{Dipole}=0.2609814,0.1112424$, $0.1646758 \backslash P G=C 01 \quad[X(C 15 H 15 S 2)] \backslash \backslash @$

\section{$\mathrm{CH}_{3} \mathrm{SC} \cdot\left(\mathrm{CH}_{2} \mathrm{Ph}\right) \mathrm{S}-\mathrm{CH}_{2} \mathrm{COOCH}_{3}(16)$}

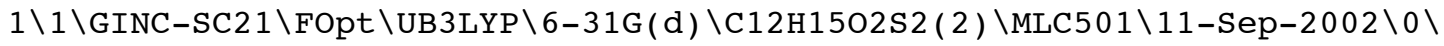
$\backslash \# \mathrm{~N}$ UB3LYP/6-31G(D) $\mathrm{SCF}=(\mathrm{TIGHT}, \mathrm{MAXCYC}=100) \quad \mathrm{OPT}=(\mathrm{MAXCYC}=100) \quad \mathrm{FREQ}$ MAXDI $\mathrm{SK}=131072000 \mathrm{GEOM}=\mathrm{CHECK} \backslash \backslash \mathrm{RRAD}(\mathrm{Z}=\mathrm{Bz} \mathrm{R}=\mathrm{MA}) \quad \mathrm{C} 2 \mathrm{D} 2=-135.1 \mathrm{D} 3=35.0 \mathrm{D} 4=100.1$ $\mathrm{D} 5=-139.9 \mathrm{D} 6=-19.0 \mathrm{D} 7=26.6 \mathrm{D} 8=-94.8 \mathrm{D} 9=146.7 \backslash \backslash 0,2 \backslash \mathrm{C}, 0.0106144613,0.63$ $94493356,0.055012908 \backslash \mathrm{S},-0.2081425176,2.3127865003,0.5580776206 \backslash \mathrm{S},-0.52$ $66335935,-0.6506483405,1.1203801117 \backslash \mathrm{C}, 0.9244422833,0.2578956307,-1.089$ $5701818 \backslash \mathrm{C},-0.9926664675,3.0543514592,-0.9314065251 \backslash \mathrm{C},-2.2996364599,-0$. $2072630242,1.425583443 \backslash \mathrm{C}, 2.2988311279,-0.2284351617,-0.6407496292 \backslash \mathrm{H}, 0$. $439953811,-0.5287777895,-1.6816927868 \backslash \mathrm{H}, 1.0450879835,1.1263495737,-1.7$ $477628181 \backslash \mathrm{C},-3.1310720782,-0.4280002157,0.1840532664 \backslash \mathrm{H},-2.3462696872,0$ $.8422373836,1.7212379072 \backslash \mathrm{H},-2.6264541194,-0.8513593961,2.2441212612 \backslash \mathrm{O}$, $-3.229974273,0.3597209174,-0.7351306789 \backslash 0,-3.7318068449,-1.6355097444$, $0.2022597776 \backslash \mathrm{C},-4.4953000781,-1.9617103961,-0.9713159759 \backslash \mathrm{H},-5.29671370$ $45,-1.2337875422,-1.1225793541 \backslash \mathrm{H},-3.8517315575,-1.971376421,-1.8550414$ $997 \backslash \mathrm{H},-4.906861465,-2.953879961,-0.7850362567 \backslash \mathrm{H},-1.9196058953,2.521199$ $7161,-1.150580256 \backslash \mathrm{H},-0.3207783111,3.0275588036,-1.7934637035 \backslash \mathrm{H},-1.2040$ $171826,4.0973387912,-0.6793988724 \backslash \mathrm{C}, 3.2655140009,0.6817168387,-0.18957$ $4798 \backslash \mathrm{C}, 2.6141358686,-1.5923566945,-0.6527862684 \backslash \mathrm{C}, 4.5160813081,0.23732$ $60476,0.2372819287 \backslash \mathrm{C}, 3.8659935778,-2.0400023896,-0.224618027 \backslash \mathrm{C}, 4.82074$ $94052,-1.1262641294,0.2215341959 \backslash \mathrm{H}, 3.0318540912,1.7434462254,-0.170465$ $3644 \backslash \mathrm{H}, 1.8744670868,-2.3087587396,-1.002646815 \backslash \mathrm{H}, 5.255342006,0.9564170$ $128,0.5808775484 \backslash \mathrm{H}, 4.0939854045,-3.1026542031,-0.243511675 \backslash \mathrm{H}, 5.7962865$ $649,-1.4720971974,0.5529201588 \backslash \backslash$ Version=DEC-AXP-OSF $/ 1-G 98 \mathrm{RevA} .11 .3 \backslash \mathrm{HF}=$ 
$-1413.0968388 \backslash \mathrm{S} 2=0.756077 \backslash \mathrm{S} 2-1=0 . \backslash \mathrm{S} 2 \mathrm{~A}=0.750027 \backslash \mathrm{RMSD}=6.211 \mathrm{e}-09 \backslash \mathrm{RMSF}=2.0$ $82 e-06 \backslash \mathrm{Dipole}=-0.5690163,-0.2843149,-0.4210236 \backslash \mathrm{PG}=\mathrm{C} 01[\mathrm{X}(\mathrm{C} 12 \mathrm{H} 15 \mathrm{O} 2 \mathrm{~S} 2)] \backslash$ 1 a

\section{$\mathrm{CH}_{3} \mathrm{SC} \cdot(\mathrm{Ph}) \mathrm{S}-\mathrm{CH}_{2} \mathrm{COOCH}_{3}(17)$}

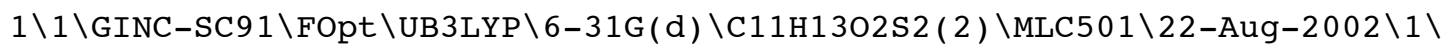
$\backslash \# N$ UB3LYP/6-31G(D) FREQ MAXDISK=131072000 FOPT $=(Z-M A T R I X, M A X C Y C=100)$ $\mathrm{SCF}=(\mathrm{QC}, \mathrm{TIGHT}, \mathrm{MAXCYC}=100) \mathrm{GEOM}=\mathrm{CHECK}$ GUESS=READ $\backslash \backslash \operatorname{RRAD}(\mathrm{Z}=\mathrm{Ph} \mathrm{R}=\mathrm{MA}) \mathrm{c} 1 \mathrm{ay} /$ $/ \mathrm{B} 3 \backslash \backslash 0,2 \backslash \mathrm{C} \backslash \mathrm{S}, 1, \mathrm{~B} 1 \backslash \mathrm{S}, 1, \mathrm{~B} 2,2, \mathrm{~A} 1 \backslash \mathrm{C}, 1, \mathrm{~B} 3,2, \mathrm{~A} 2,3, \mathrm{D} 1,0 \backslash \mathrm{C}, 2, \mathrm{~B} 4,1, \mathrm{~A} 3,3, \mathrm{D} 2,0 \backslash \mathrm{C}$, $3, \mathrm{~B} 5,1, \mathrm{~A} 4,2, \mathrm{D} 3,0 \backslash \mathrm{C}, 6, \mathrm{~B} 6,3, \mathrm{~A} 5,1, \mathrm{D} 4,0 \backslash \mathrm{H}, 6, \mathrm{~B} 7,3, \mathrm{~A} 6,1, \mathrm{D} 5,0 \backslash \mathrm{H}, 6, \mathrm{~B} 8,3, \mathrm{~A} 7,1, \mathrm{D}$ $6,0 \backslash 0,7, \mathrm{~B} 9,6, \mathrm{~A} 8,3, \mathrm{D} 7,0 \backslash 0,7, \mathrm{~B} 10,6, \mathrm{~A} 9,10, \mathrm{D} 8,0 \backslash \mathrm{C}, 11, \mathrm{~B} 11,7, \mathrm{~A} 10,6, \mathrm{D} 9,0 \backslash \mathrm{H}, 12$

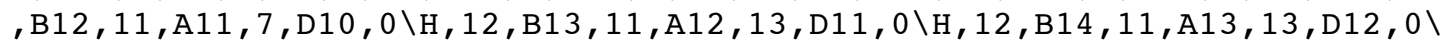
$\mathrm{H}, 5, \mathrm{~B} 15,2, \mathrm{~A} 14,1, \mathrm{D} 13,0 \backslash \mathrm{H}, 5, \mathrm{~B} 16,2, \mathrm{~A} 15,16, \mathrm{D} 14,0 \backslash \mathrm{H}, 5, \mathrm{~B} 17,2, \mathrm{~A} 16,16, \mathrm{D} 15,0 \backslash \mathrm{C}$, $4, \mathrm{~B} 18,1, \mathrm{~A} 17,2, \mathrm{D} 16,0 \backslash \mathrm{C}, 4, \mathrm{~B} 19,1, \mathrm{~A} 18,19, \mathrm{D} 17,0 \backslash \mathrm{C}, 19, \mathrm{~B} 20,4, \mathrm{~A} 19,1, \mathrm{D} 18,0 \backslash \mathrm{C}, 20$ $, \mathrm{B} 21,4, \mathrm{~A} 20,1, \mathrm{D} 19,0 \backslash \mathrm{C}, 21, \mathrm{~B} 22,19, \mathrm{~A} 21,4, \mathrm{D} 20,0 \backslash \mathrm{H}, 19, \mathrm{~B} 23,4, \mathrm{~A} 22,21, \mathrm{D} 21,0 \backslash \mathrm{H}, 2$ $0, \mathrm{~B} 24,4, \mathrm{~A} 23,22, \mathrm{D} 22,0 \backslash \mathrm{H}, 21, \mathrm{~B} 25,19, \mathrm{~A} 24,23, \mathrm{D} 23,0 \backslash \mathrm{H}, 22, \mathrm{~B} 26,20, \mathrm{~A} 25,4, \mathrm{D} 24,0 \backslash$ $\mathrm{H}, 23, \mathrm{~B} 27,21, \mathrm{~A} 26,19, \mathrm{D} 25,0 \backslash \backslash \mathrm{D} 2=-48.66742221 \backslash \mathrm{D} 3=-59.98700545 \backslash \mathrm{D} 5=159.47267$ $01 \backslash D 4=-80.35076392 \backslash D 6=43.40237843 \backslash D 7=-8.87674609 \backslash B 1=1.76954697 \backslash B 2=1.77$ $231178 \backslash \mathrm{B} 3=1.44369491 \backslash \mathrm{B} 4=1.83547449 \backslash \mathrm{B} 5=1.83134785 \backslash \mathrm{B} 6=1.52496927 \backslash \mathrm{B} 7=1.09$ $587204 \backslash \mathrm{B} 8=1.0936977 \backslash \mathrm{B} 9=1.2088986 \backslash \mathrm{B} 10=1.35071877 \backslash \mathrm{B} 11=1.4390682 \backslash \mathrm{B} 12=1.09$ $317885 \backslash \mathrm{B} 13=1.09020781 \backslash \mathrm{B} 14=1.09294778 \backslash \mathrm{B} 15=1.0933617 \backslash \mathrm{B} 16=1.09122537 \backslash \mathrm{B} 17=$ $1.09255848 \backslash \mathrm{B} 18=1.42267223 \backslash \mathrm{B} 19=1.4224648 \backslash \mathrm{B} 20=1.38817705 \backslash \mathrm{B} 21=1.38816973 \backslash$ $\mathrm{B} 22=1.39949408 \backslash \mathrm{B} 23=1.08353903 \backslash \mathrm{B} 24=1.08443776 \backslash \mathrm{B} 25=1.08696969 \backslash \mathrm{B} 26=1.0870$ $7355 \backslash \mathrm{B} 27=1.08653262 \backslash \mathrm{A} 1=119.35031129 \backslash \mathrm{A} 2=120.11353834 \backslash \mathrm{A} 3=104.00387848 \backslash \mathrm{A} 4$ $=103.03887394 \backslash A 5=114.56529937 \backslash A 6=106.75033321 \backslash A 7=110.06765083 \backslash A 8=127.0$ $2742896 \backslash A 9=109.08604933 \backslash A 10=115.35654399 \backslash A 11=110.51804799 \backslash A 12=105.5020$ $0456 \backslash A 13=110.43056808 \backslash A 14=106.05484832 \backslash A 15=111.04466808 \backslash A 16=110.586636$ $44 \backslash A 17=121.17625633 \backslash A 18=121.49310815 \backslash A 19=120.9094988 \backslash A 20=121.10236351 \backslash$ $\mathrm{A} 21=120.81033128 \backslash \mathrm{A} 22=118.91779171 \backslash \mathrm{A} 23=119.08284007 \backslash \mathrm{A} 24=119.22802087 \backslash \mathrm{A} 2$ $5=119.36384162 \backslash A 26=120.41384942 \backslash D 1=180.23211003 \backslash D 8=-178.9984738 \backslash D 9=179$ $.00140925 \backslash \mathrm{D} 10=-60.56985178 \backslash \mathrm{D} 11=-119.64827693 \backslash \mathrm{D} 12=120.73334999 \backslash \mathrm{D} 13=174$. $05251161 \backslash D 14=-119.06185435 \backslash D 15=118.72690141 \backslash D 16=-201.96568734 \backslash D 17=-180$ $.04187775 \backslash \mathrm{D} 18=-180.56294225 \backslash \mathrm{D} 19=180.5191603 \backslash \mathrm{D} 20=0.13941807 \backslash \mathrm{D} 21=177.506$ $21699 \backslash \mathrm{D} 22=178.84814744 \backslash \mathrm{D} 23=179.23254714 \backslash \mathrm{D} 24=179.36224919 \backslash \mathrm{D} 25=-179.9396$ $5887 \backslash \backslash$ Version=DEC-AXP-OSF $/ 1-G 98$ RevA. $11.3 \backslash \mathrm{HF}=-1373.7927754 \backslash \mathrm{S} 2=0.772138 \backslash$ $\mathrm{S} 2-1=0 . \backslash \mathrm{S} 2 \mathrm{~A}=0.750363 \backslash \mathrm{RMSD}=0.000 \mathrm{e}+00 \backslash \mathrm{RMSF}=8.625 \mathrm{e}-05 \backslash \mathrm{Dipole}=0.5647553,0$. $3523014,0.5202045 \backslash \mathrm{PG}=\mathrm{C} 01 \quad[\mathrm{X}(\mathrm{C} 11 \mathrm{H} 1302 \mathrm{~S} 2)] \backslash \backslash @$

\section{$\mathrm{CH}_{3} \mathrm{SC} \cdot\left(\mathrm{CH}_{2} \mathrm{Ph}\right) \mathrm{S}-\mathrm{C}\left(\mathrm{CH}_{3}\right)_{2} \mathrm{CN}(18)$}

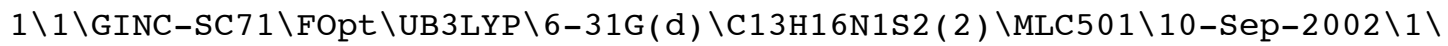
$\backslash \# \mathrm{~N}$ UB3LYP/6-31G(D) OPT $=(\mathrm{Z}-\mathrm{MATRIX}, \mathrm{MAXCYC}=100)$ FREQ MAXDISK=131072000 S $\mathrm{CF}=(\mathrm{TIGHT}, \mathrm{MAXCYC}=100) \quad \mathrm{INT}(\mathrm{GRID}=\mathrm{ULTRAFINE}) \quad \mathrm{GEOM}=\mathrm{CHECK} \backslash \backslash \mathrm{RRAD}(\mathrm{Z}=\mathrm{BZ} \mathrm{R}=\mathrm{MCN})$ $\mathrm{C} 1 \backslash \backslash 0,2 \backslash \mathrm{C} \backslash \mathrm{S}, 1, \mathrm{~B} 1 \backslash \mathrm{S}, 1, \mathrm{~B} 2,2, \mathrm{~A} 1 \backslash \mathrm{C}, 1, \mathrm{~B} 3,2, \mathrm{~A} 2,3, \mathrm{D} 1,0 \backslash \mathrm{C}, 4, \mathrm{~B} 4,1, \mathrm{~A} 3,2, \mathrm{D} 2,0 \backslash \mathrm{H}$, $4, \mathrm{~B} 5,1, \mathrm{~A} 4,2, \mathrm{D} 3,0 \backslash \mathrm{H}, 4, \mathrm{~B} 6,1, \mathrm{~A} 5,2, \mathrm{D} 4,0 \backslash \mathrm{C}, 3, \mathrm{~B} 7,1, \mathrm{~A} 6,2, \mathrm{D} 5,0 \backslash \mathrm{C}, 2, \mathrm{~B} 8,1, \mathrm{~A} 7,3, \mathrm{D}$ $6,0 \backslash \mathrm{C}, 9, \mathrm{~B} 9,2, \mathrm{~A} 8,1, \mathrm{D} 7,0 \backslash \mathrm{C}, 9, \mathrm{~B} 10,2, \mathrm{~A} 9,1, \mathrm{D} 8,0 \backslash \mathrm{C}, 9, \mathrm{~B} 11,2, \mathrm{~A} 10,1, \mathrm{D} 9,0 \backslash \mathrm{H}, 11, \mathrm{~B}$ $12,9, \mathrm{~A} 11,2, \mathrm{D} 10,0 \backslash \mathrm{H}, 11, \mathrm{~B} 13,9, \mathrm{~A} 12,13, \mathrm{D} 11,0 \backslash \mathrm{H}, 11, \mathrm{~B} 14,9, \mathrm{~A} 13,13, \mathrm{D} 12,0 \backslash \mathrm{H}, 8, \mathrm{~B}$ $15,3, \mathrm{~A} 14,1, \mathrm{D} 13,0 \backslash \mathrm{H}, 8, \mathrm{~B} 16,3, \mathrm{~A} 15,16, \mathrm{D} 14,0 \backslash \mathrm{H}, 8, \mathrm{~B} 17,3, \mathrm{~A} 16,16, \mathrm{D} 15,0 \backslash \mathrm{C}, 5, \mathrm{~B} 18$ , 4, A17, 1, D16, 0\C , 19 , B19, $5, \mathrm{~A} 18,4, \mathrm{D} 17,0 \backslash \mathrm{C}, 20, \mathrm{~B} 20,19, \mathrm{~A} 19,5, \mathrm{D} 18,0 \backslash \mathrm{C}, 21, \mathrm{~B} 21$ , $20, \mathrm{~A} 20,19, \mathrm{D} 19,0 \backslash \mathrm{C}, 22, \mathrm{~B} 22,21, \mathrm{~A} 21,20, \mathrm{D} 20,0 \backslash \mathrm{H}, 23, \mathrm{~B} 23,22, \mathrm{~A} 22,21, \mathrm{D} 21,0 \backslash \mathrm{H}, 2$ 2 , B2 4, 21, A2 3, $23, \mathrm{D} 22,0 \backslash \mathrm{H}, 21, \mathrm{~B} 25,20, \mathrm{~A} 24,22, \mathrm{D} 23,0 \backslash \mathrm{H}, 20, \mathrm{~B} 26,19, \mathrm{~A} 25,21, \mathrm{D} 24$, $0 \backslash \mathrm{H}, 19, \mathrm{~B} 27,5, \mathrm{~A} 26,20, \mathrm{D} 25,0 \backslash \mathrm{H}, 10, \mathrm{~B} 28,9, \mathrm{~A} 27,2, \mathrm{D} 26,0 \backslash \mathrm{H}, 10, \mathrm{~B} 29,9, \mathrm{~A} 28,29, \mathrm{D} 27$ , $0 \backslash \mathrm{H}, 10, \mathrm{~B} 30,9, \mathrm{~A} 29,29, \mathrm{D} 28,0 \backslash \mathrm{X}, 12,1 ., 9,90.2, \mathrm{D} 29,0 \backslash \mathrm{N}, 12, \mathrm{~B} 31,32, \mathrm{~A} 30,9,180$ ., $0 \backslash \backslash \mathrm{B} 1=1.74158315 \backslash \mathrm{B} 2=1.75854282 \backslash \mathrm{B} 3=1.51907585 \backslash \mathrm{B} 4=1.52268081 \backslash \mathrm{B} 5=1.0958$ $8909 \backslash B 6=1.09817176 \backslash B 7=1.82271722 \backslash B 8=1.92209999 \backslash B 9=1.53935692 \backslash B 10=1.534$ $22373 \backslash B 11=1.46613269 \backslash B 12=1.09437876 \backslash B 13=1.09651774 \backslash B 14=1.09314929 \backslash B 15=$ $1.09224159 \backslash \mathrm{B} 16=1.09145599 \backslash \mathrm{B} 17=1.09324982 \backslash \mathrm{B} 18=1.39957428 \backslash \mathrm{B} 19=1.39671712$ $\backslash \mathrm{B} 20=1.39507851 \backslash \mathrm{B} 21=1.39728989 \backslash \mathrm{B} 22=1.39428744 \backslash \mathrm{B} 23=1.08779339 \backslash \mathrm{B} 24=1.087$ $11536 \backslash \mathrm{B} 25=1.0868793 \backslash \mathrm{B} 26=1.08697623 \backslash \mathrm{B} 27=1.08719939 \backslash \mathrm{B} 28=1.09372247 \backslash \mathrm{B} 29=1$ 
$.09542121 \backslash \mathrm{B} 30=1.09431937 \backslash \mathrm{B} 31=1.16285746 \backslash \mathrm{A} 1=123.71423017 \backslash \mathrm{A} 2=121.8675341$ $1 \backslash A 3=113.0507604 \backslash A 4=109.65077295 \backslash A 5=108.81848364 \backslash A 6=105.50539826 \backslash A 7=10$ $6.73616068 \backslash A 8=104.45253669 \backslash A 9=111.2714673 \backslash A 10=108.42077149 \backslash A 11=109.517$ $10323 \backslash A 12=110.19190686 \backslash A 13=111.40642512 \backslash A 14=110.865312 \backslash A 15=111.3486486$ $7 \backslash A 16=105.65278598 \backslash A 17=120.54947403 \backslash A 18=120.55011096 \backslash A 19=120.19384831 \backslash$ $\mathrm{A} 20=119.62352595 \backslash \mathrm{A} 21=120.11929502 \backslash \mathrm{A} 22=119.85626635 \backslash \mathrm{A} 23=120.08796574 \backslash \mathrm{A} 2$ $4=120.22372982 \backslash \mathrm{A} 25=119.67165151 \backslash \mathrm{A} 26=119.43012822 \backslash \mathrm{A} 27=111.38138115 \backslash \mathrm{A} 28=$ $109.91828511 \backslash \mathrm{A} 29=109.65802699 \backslash \mathrm{A} 30=89.86785275 \backslash \mathrm{D} 1=178.40067204 \backslash \mathrm{D} 2=-66.2$ $9532724 \backslash D 3=55.67399414 \backslash D 4=171.76229967 \backslash D 5=10.15203796 \backslash D 6=102.57985811 \backslash$ $\mathrm{D} 7=-174.95979184 \backslash \mathrm{D} 8=-53.78971269 \backslash \mathrm{D} 9=67.95881995 \backslash \mathrm{D} 10=-61.30028854 \backslash \mathrm{D} 11=-$ $119.3555044 \backslash D 12=120.32545641 \backslash D 13=60.92871766 \backslash D 14=-122.35888935 \backslash D 15=118$ $.67387775 \backslash D 16=106.21187794 \backslash D 17=-179.76621529 \backslash D 18=0.02519765 \backslash D 19=0.0161$ $3592 \backslash \mathrm{D} 20=0.04305114 \backslash \mathrm{D} 21=-180.02200919 \backslash \mathrm{D} 22=-179.79744188 \backslash \mathrm{D} 23=180.146764$ $69 \backslash D 24=-179.85770283 \backslash D 25=180.0763185 \backslash D 26=-58.02109584 \backslash D 27=-120.1186253$ $1 \backslash \mathrm{D} 28=120.5100964 \backslash \mathrm{D} 29=-175.97427552 \backslash \backslash$ Version=DEC-AXP-OSF $/ 1-G 98 \mathrm{RevA} .11$. $3 \backslash \mathrm{HF}=-1356.0904085 \backslash \mathrm{S} 2=0.756062 \backslash \mathrm{S} 2-1=0 . \backslash \mathrm{S} 2 \mathrm{~A}=0.750028 \backslash \mathrm{RMSD}=8.122 \mathrm{e}-09 \backslash \mathrm{RMS}$ $\mathrm{F}=2.193 e-05 \backslash \mathrm{Dipole}=1.6064184,0.0385489,0.4576216 \backslash \mathrm{PG}=\mathrm{C} 01 \quad[\mathrm{X}(\mathrm{C} 13 \mathrm{H} 16 \mathrm{~N} 1 \mathrm{~S} 2)$ ] $\backslash \backslash @$

\section{$\mathrm{CH}_{3} \mathrm{SC} \cdot(\mathrm{Ph}) \mathrm{S}-\mathrm{C}\left(\mathrm{CH}_{3}\right)_{2} \mathrm{CN}(19)$}

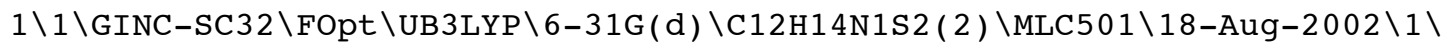
$\backslash \# \mathrm{~N}$ UB3LYP/6-31G(D) FREQ MAXDISK=131072000 FOPT $=(\mathrm{Z}-\mathrm{MATRIX}, \mathrm{MAXCYC}=100)$ $\mathrm{SCF}=(\mathrm{QC}, \mathrm{TIGHT}, \mathrm{MAXCYC}=100) \backslash \backslash \mathrm{RRAD}(\mathrm{Z}=\mathrm{Ph} \mathrm{R}=\mathrm{MCN}) \mathrm{C} 1 \mathrm{ax} / / \mathrm{B} 3 \backslash \backslash 0,2 \backslash \mathrm{C} \backslash \mathrm{S}, 1, \mathrm{~B} 1 \backslash \mathrm{S}, 1$ , $\mathrm{B} 2,2, \mathrm{~A} 1 \backslash \mathrm{C}, 1, \mathrm{~B} 3,2, \mathrm{~A} 2,3, \mathrm{D} 1,0 \backslash \mathrm{C}, 3, \mathrm{~B} 4,1, \mathrm{~A} 3,2, \mathrm{D} 2,0 \backslash \mathrm{C}, 2, \mathrm{~B} 5,1, \mathrm{~A} 4,3, \mathrm{D} 3,0 \backslash \mathrm{C}, 6$, $\mathrm{B} 6,2, \mathrm{~A} 5,1, \mathrm{D} 4,0 \backslash \mathrm{C}, 6, \mathrm{~B} 7,2, \mathrm{~A} 6,1, \mathrm{D} 5,0 \backslash \mathrm{C}, 6, \mathrm{~B} 8,2, \mathrm{~A} 7,1, \mathrm{D} 6,0 \backslash \mathrm{H}, 8, \mathrm{~B} 9,6, \mathrm{~A} 8,2, \mathrm{D} 7$, $0 \backslash \mathrm{H}, 8, \mathrm{~B} 10,6, \mathrm{~A} 9,10, \mathrm{D} 8,0 \backslash \mathrm{H}, 8, \mathrm{~B} 11,6, \mathrm{~A} 10,10, \mathrm{D} 9,0 \backslash \mathrm{H}, 5, \mathrm{~B} 12,3, \mathrm{~A} 11,1, \mathrm{D} 10,0 \backslash \mathrm{H}, 5$ , $\mathrm{B} 13,3, \mathrm{~A} 12,13, \mathrm{D} 11,0 \backslash \mathrm{H}, 5, \mathrm{~B} 14,3, \mathrm{~A} 13,13, \mathrm{D} 12,0 \backslash \mathrm{C}, 4, \mathrm{~B} 15,1, \mathrm{~A} 14,2, \mathrm{D} 13,0 \backslash \mathrm{C}, 4, \mathrm{~B}$ $16,1, \mathrm{~A} 15,16, \mathrm{D} 14,0 \backslash \mathrm{C}, 16, \mathrm{~B} 17,4, \mathrm{~A} 16,1, \mathrm{D} 15,0 \backslash \mathrm{C}, 17, \mathrm{~B} 18,4, \mathrm{~A} 17,1, \mathrm{D} 16,0 \backslash \mathrm{C}, 18, \mathrm{~B}$ $19,16, \mathrm{~A} 18,4, \mathrm{D} 17,0 \backslash \mathrm{H}, 16, \mathrm{~B} 20,4, \mathrm{~A} 19,18, \mathrm{D} 18,0 \backslash \mathrm{H}, 17, \mathrm{~B} 21,4, \mathrm{~A} 20,19, \mathrm{D} 19,0 \backslash \mathrm{H}, 18$ , B22 , 16, A21 , $20, \mathrm{D} 20,0 \backslash \mathrm{H}, 19, \mathrm{~B} 23,17, \mathrm{~A} 22,4, \mathrm{D} 21,0 \backslash \mathrm{H}, 20, \mathrm{~B} 24,18, \mathrm{~A} 23,16, \mathrm{D} 22,0 \backslash$ $\mathrm{H}, 9, \mathrm{~B} 25,6, \mathrm{~A} 24,2, \mathrm{D} 23,0 \backslash \mathrm{H}, 9, \mathrm{~B} 26,6, \mathrm{~A} 25,26, \mathrm{D} 24,0 \backslash \mathrm{H}, 9, \mathrm{~B} 27,6, \mathrm{~A} 26,26, \mathrm{D} 25,0 \backslash \mathrm{X}$, $7,1 ., 6,90 ., 2, \mathrm{D} 26,0 \backslash \mathrm{N}, 7, \mathrm{~B} 28,29, \mathrm{~A} 27,6,180 ., 0 \backslash \backslash \mathrm{D} 2=-35.09021444 \backslash \mathrm{D} 3=-70.940$ $85775 \backslash D 6=197.13342316 \backslash D 5=-41.88397446 \backslash D 4=80.53324022 \backslash B 1=1.76266592 \backslash B 2=$ $1.76491418 \backslash \mathrm{B} 3=1.45038859 \backslash \mathrm{B} 4=1.83068375 \backslash \mathrm{B} 5=1.90935687 \backslash \mathrm{B} 6=1.46759559 \backslash \mathrm{B} 7=$ $1.53462562 \backslash B 8=1.54173988 \backslash B 9=1.09360837 \backslash B 10=1.09238336 \backslash B 11=1.09614805 \backslash B$ $12=1.08974979 \backslash \mathrm{B} 13=1.09349872 \backslash \mathrm{B} 14=1.09169781 \backslash \mathrm{B} 15=1.42135308 \backslash \mathrm{B} 16=1.42224$ $621 \backslash \mathrm{B} 17=1.38833895 \backslash \mathrm{B} 18=1.38875137 \backslash \mathrm{B} 19=1.39890536 \backslash \mathrm{B} 20=1.08377272 \backslash \mathrm{B} 21=1$. $0841309 \backslash \mathrm{B} 22=1.08699759 \backslash \mathrm{B} 23=1.08689321 \backslash \mathrm{B} 24=1.08627917 \backslash \mathrm{B} 25=1.09387261 \backslash \mathrm{B} 2$ $6=1.09408458 \backslash \mathrm{B} 27=1.09534711 \backslash \mathrm{B} 28=1.16249426 \backslash \mathrm{A} 1=120.64780033 \backslash \mathrm{A} 2=120.8256$ $1796 \backslash A 3=104.97948192 \backslash A 4=106.61983892 \backslash A 5=109.1352656 \backslash A 6=111.64536538 \backslash A 7$ $=104.37277628 \backslash \mathrm{A} 8=109.71362489 \backslash \mathrm{A} 9=111.42341574 \backslash \mathrm{A} 10=109.70032951 \backslash \mathrm{A} 11=111$ $.61813326 \backslash A 12=104.93397596 \backslash A 13=110.39152846 \backslash A 14=121.78396563 \backslash A 15=121.3$ $6258547 \backslash A 16=121.33987705 \backslash A 17=121.30614498 \backslash A 18=120.72823875 \backslash A 19=119.062$ $07754 \backslash \mathrm{A} 20=119.30540768 \backslash \mathrm{A} 21=119.28627841 \backslash \mathrm{A} 22=119.24848809 \backslash \mathrm{A} 23=120.47331$ $105 \backslash A 24=109.67113877 \backslash A 25=111.44932392 \backslash A 26=109.78128488 \backslash A 27=88.66365939$ $\backslash D 1=-181.79082602 \backslash D 7=-59.51250006 \backslash D 8=120.55744976 \backslash D 9=-119.41849859 \backslash D 10$ $=72.18191784 \backslash \mathrm{D} 11=118.21694492 \backslash \mathrm{D} 12=-123.83462359 \backslash \mathrm{D} 13=-13.94994726 \backslash \mathrm{D} 14=1$ $79.91949005 \backslash D 15=180.52028983 \backslash D 16=-180.83850649 \backslash D 17=0.08703076 \backslash D 18=-179$ $.88768344 \backslash \mathrm{D} 19=-181.18633615 \backslash \mathrm{D} 20=-180.19903579 \backslash \mathrm{D} 21=-180.15406071 \backslash \mathrm{D} 22=17$ $9.92619888 \backslash \mathrm{D} 23=64.07024737 \backslash \mathrm{D} 24=-120.59077904 \backslash \mathrm{D} 25=119.51217784 \backslash \mathrm{D} 26=-226$ $.78647794 \backslash \backslash$ Version=DEC-AXP-OSF/1-G98RevA. $11.3 \backslash \mathrm{HF}=-1316.7874212 \backslash \mathrm{S} 2=0.77$ $0033 \backslash \mathrm{S} 2-1=0 . \backslash \mathrm{S} 2 \mathrm{~A}=0.750307 \backslash \mathrm{RMSD}=0.000 \mathrm{e}+00 \backslash \mathrm{RMSF}=6.792 \mathrm{e}-05 \backslash \mathrm{Dipole}=-1.1619$ $908,-0.0511278,0.0690447 \backslash \mathrm{PG}=\mathrm{C} 01 \quad[\mathrm{X}(\mathrm{C} 12 \mathrm{H} 14 \mathrm{~N} 1 \mathrm{~S} 2)] \backslash \backslash @$

\section{$\mathrm{H}-\mathrm{CH}_{3}$}

$1 \backslash 1 \backslash$ GINC-RSCQC2 $\backslash$ FOpt $\backslash R B 3 L Y P \backslash 6-31 G(d) \backslash C 1 H 4 \backslash M I C H E L L E \backslash 01-J u n-2001 \backslash 1 \backslash \backslash \# P R$ B3LYP/6-31G* OPT=Z-MATRIX FREQ=NORAMAN MAXDISK=262144000\\METHANE TEST $\backslash \backslash 0,1 \backslash \mathrm{C} \backslash \mathrm{H}, 1, \mathrm{bl} \backslash \mathrm{H}, 1, \mathrm{bl}, 2,109.47122063 \backslash \mathrm{H}, 1, \mathrm{bl}, 2,109.47122063,3,120 ., 0 \backslash \mathrm{H}$, 
$1, \mathrm{bl}, 2,109.47122063,3,-120 ., 0 \backslash \backslash \mathrm{bl}=1.09326575 \backslash \backslash$ Version=IBM-RS6000-G98Re $\mathrm{VA} .9 \backslash \mathrm{HF}=-40.5183892 \backslash \mathrm{RMSD}=1.077 \mathrm{e}-09 \backslash \mathrm{RMSF}=1.309 \mathrm{e}-08 \backslash \mathrm{Dipole}=0 ., 0 ., 0 . \backslash \mathrm{PG}=\mathrm{T}$ D $[\mathrm{O}(\mathrm{C} 1), 4 \mathrm{C} 3(\mathrm{H} 1)] \backslash \backslash \mathrm{Q}$

\section{$\mathrm{H}-\mathrm{CH}_{2} \mathrm{Ph}$}

$1 \backslash 1 \backslash G I N C-L C 27 \backslash F O p t \backslash R B 3 L Y P \backslash 6-31 G(d) \backslash C 7 H 8 \backslash M L C 501 \backslash 16-M a r-2004 \backslash 0 \backslash \backslash \#$ B3LYP/ 6-31G* SCF=TIGHT OPT=TIGHT FREQ MAXDISK $=402653184 \backslash \backslash \mathrm{CH} 3 \mathrm{Ph} \backslash \backslash 0,1 \backslash \mathrm{H},-0.012$ $280233,0.7346071951,2.1468279811 \backslash \mathrm{C},-0.0074819681,0.1943288714,1.202363$ $6156 \backslash C,-0.0067265807,-1.2011662732,1.2054029939 \backslash C,-0.0056582472,-1.905$ $0507128,0 . \backslash C,-0.0067265807,-1.2011662732,-1.2054029939 \backslash C,-0.0074819681$ $, 0.1943288714,-1.2023636156 \backslash \mathrm{C},-0.004493163,0.9139230304,0 . \backslash \mathrm{C}, 0.0272590$ $169,2.4252659094,0 . \backslash \mathrm{H}, 1.0589780721,2.8022373154,0 . \backslash \mathrm{H},-0.4676659209,2.8$ $373136412,0.8860302766 \backslash \mathrm{H},-0.4676659209,2.8373136412,-0.8860302766 \backslash \mathrm{H},-0$ $.012280233,0.7346071951,-2.1468279811 \backslash \mathrm{H},-0.0114411572,-1.7385214103,-2$ $.1504319414 \backslash \mathrm{H},-0.0083465047,-2.9918167074,0 . \backslash \mathrm{H},-0.0114411572,-1.738521$ $4103,2.1504319414 \backslash \backslash$ Version=x86-Linux-G03RevB.03\State $=1-A^{\prime} \backslash H F=-271.566$ $6505 \backslash \mathrm{RMSD}=8.405 \mathrm{e}-09 \backslash \mathrm{RMSF}=1.362 \mathrm{e}-06 \backslash \mathrm{Dipole}=0.0152484,0.1248415,0 . \backslash \mathrm{PG}=\mathrm{CS}$

$[\mathrm{SG}(\mathrm{C} 3 \mathrm{H} 2), \mathrm{X}(\mathrm{C} 4 \mathrm{H} 6)] \backslash \backslash @$

\section{$\mathrm{H}-\mathrm{CH}_{2} \mathrm{COOCH}_{3}$}

$1 \backslash 1 \backslash G I N C-S C 105 \backslash F O p t \backslash R B 3 L Y P \backslash 6-31 G(d) \backslash C 3 H 602 \backslash M L C 501 \backslash 16-M a r-2004 \backslash 0 \backslash \backslash \#$ B3L YP/6-31G* OPT FREQ MAXDISK=65536000\\ch3cooch3-geom-6dub3 $\backslash \backslash 0,1 \backslash \mathrm{C}, 0 ., 0$. , $0 . \backslash 0,1.1806641117,0.2693571148,0 . \backslash 0,-0.4766353163,-1.2676815605,0 . \backslash \mathrm{C}$, $0.5238438782,-2.2984404094,0 . \backslash \mathrm{C},-1.1338518662,0.9987105655,0 . \backslash \mathrm{H},-0.726$ $1641654,2.0099935722,0 . \backslash \mathrm{H},-0.0257634362,-3.2403316547,0 . \backslash \mathrm{H}, 1.155560253$ $1,-2.2224675233,0.88922 \backslash \mathrm{H}, 1.1555602531,-2.2224675233,-0.88922 \backslash \mathrm{H},-1.765$ $6876699,0.851506761,-0.88217 \backslash \mathrm{H},-1.7656876699,0.851506761,0.88217 \backslash \backslash$ Vers ion=DEC-AXP-OSF /1-G03RevB .03 \State $=1-A^{\prime} \backslash \mathrm{HF}=-268.3884834 \backslash \mathrm{RMSD}=2.990 \mathrm{e}-09$ $\backslash \mathrm{RMSF}=3.448 \mathrm{e}-05 \backslash \mathrm{Dipole}=-0.6571523,0.2409099,0 . \backslash \mathrm{PG}=\mathrm{CS} \quad[\mathrm{SG}(\mathrm{C} 3 \mathrm{H} 2 \mathrm{O} 2), \mathrm{X}(\mathrm{H} 4)$ ] \\@

\section{$\mathrm{H}-\mathrm{C}\left(\mathrm{CH}_{3}\right)_{2} \mathrm{CN}$}

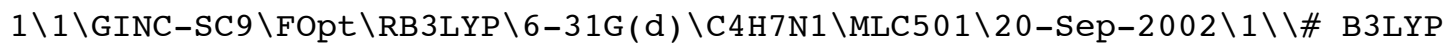

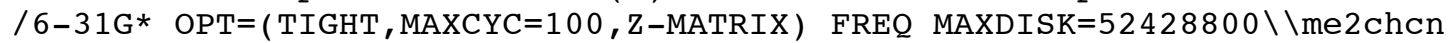
$-\mathrm{Cs}-6$ dub3 $\backslash \backslash 0,1 \backslash \mathrm{C} \backslash \mathrm{C}, 1, \mathrm{~B} 1 \backslash \mathrm{X}, 2,1 ., 1,90 . \backslash \mathrm{N}, 2, \mathrm{~B} 2,3, \mathrm{~A} 1,1,180 ., 0 \backslash \mathrm{C}, 1, \mathrm{~B} 3,2, \mathrm{~A} 2$, $4, \mathrm{D} 1,0 \backslash \mathrm{C}, 1, \mathrm{~B} 3,2, \mathrm{~A} 2,4,-\mathrm{D} 1,0 \backslash \mathrm{H}, 5, \mathrm{~B} 4,1, \mathrm{~A} 3,2, \mathrm{D} 2,0 \backslash \mathrm{H}, 6, \mathrm{~B} 4,1, \mathrm{~A} 3,2,-\mathrm{D} 2,0 \backslash \mathrm{H}, 5$, $\mathrm{B} 5,1, \mathrm{~A} 4,7, \mathrm{D} 3,0 \backslash \mathrm{H}, 5, \mathrm{~B} 6,1, \mathrm{~A} 5,7, \mathrm{D} 4,0 \backslash \mathrm{H}, 6, \mathrm{~B} 6,1, \mathrm{~A} 5,8,-\mathrm{D} 4,0 \backslash \mathrm{H}, 6, \mathrm{~B} 5,1, \mathrm{~A} 4,8,-\mathrm{D}$ $3,0 \backslash \mathrm{H}, 1, \mathrm{~B} 7,2, \mathrm{~A} 6,4,180 ., 0 \backslash \backslash \mathrm{B} 7=1.09858521 \backslash \mathrm{A} 6=106.60432393 \backslash \mathrm{B} 1=1.47251393 \backslash$ $\mathrm{B} 2=1.16116373 \backslash \mathrm{B} 3=1.54180066 \backslash \mathrm{B} 4=1.09489716 \backslash \mathrm{B} 5=1.09399754 \backslash \mathrm{B} 6=1.0950837 \backslash \mathrm{A}$ $1=89.39524564 \backslash A 2=110.46001989 \backslash A 3=110.73492571 \backslash A 4=111.34414552 \backslash A 5=109.5$ $9179039 \backslash \mathrm{D} 1=62.34809296 \backslash \mathrm{D} 2=296.77899993 \backslash \mathrm{D} 3=120.52082383 \backslash \mathrm{D} 4=-119.4655672$ $4 \backslash \backslash$ Version=DEC-AXP-OSF / 1-G98RevA.11.3 S State $=1-A^{\prime} \backslash H F=-211.3831069 \backslash R M S D=$ $5.940 \mathrm{e}-09 \backslash \mathrm{RMSF}=3.751 \mathrm{e}-06 \backslash \mathrm{Dipole}=0.0514549,0,,-1.550366 \backslash \mathrm{PG}=\mathrm{CS} \quad[\mathrm{SG}(\mathrm{C} 2 \mathrm{H} 1 \mathrm{~N}$ $1), \mathrm{X}(\mathrm{C} 2 \mathrm{H} 6) \mathrm{]} \backslash \backslash \mathrm{Q}$

\section{$\cdot \mathrm{CH}_{2} \mathrm{CH}_{3}$}

$1 \backslash 1 \backslash G I N C-S C 116 \backslash F O p t \backslash U B 3 L Y P \backslash 6-31 G(d) \backslash C 2 H 5(2) \backslash M L C 501 \backslash 06-M a r-2003 \backslash 0 \backslash \backslash \# N B$ 3LYP/6-31G(D) OPT=TIGHT FREQ MAXDISK=13107200\\Et_rad Cs $\backslash \backslash 0,2 \backslash C,-0.001$ $4599287,0.7954773663,0 . \backslash \mathrm{C}, 0.0222526364,-0.6939020 \overline{5} 91,0 . \backslash \mathrm{H},-0.994810722$ $3,-1.1263631861,0 . \backslash \mathrm{H},-0.0929966331,1.3519648812,0.9274633127 \backslash \mathrm{H},-0.0929$ $966331,1.3519648812,-0.9274633127 \backslash \mathrm{H}, 0.5280238711,-1.0935092097,0.88764$ $34386 \backslash \mathrm{H}, 0.5280238711,-1.0935092097,-0.8876434386 \backslash \backslash$ Version=DEC-AXP-OSF / 1-G98RevA. 11.3 \State=2-A ${ }^{\prime} \backslash \mathrm{HF}=-79.1578673 \backslash \mathrm{S} 2=0.753921 \backslash \mathrm{S} 2-1=0 . \backslash \mathrm{S} 2 \mathrm{~A}=0.750$ $01 \backslash \mathrm{RMSD}=8.620 \mathrm{e}-09 \backslash \mathrm{RMSF}=3.177 \mathrm{e}-06 \backslash \mathrm{Dipole}=-0.0504824,-0.0860887,0 . \backslash \mathrm{PG}=\mathrm{CS}$ $[\mathrm{SG}(\mathrm{C} 2 \mathrm{H} 1), \mathrm{X}(\mathrm{H} 4)] \backslash \backslash @$

\section{$\mathrm{H}-\mathrm{CH}_{2} \mathrm{CH}_{3}$}

$1 \backslash 1 \backslash G I N C-S C 4 \backslash F O p t \backslash R B 3 L Y P \backslash 6-31 G(d) \backslash C 2 H 6 \backslash M L C 501 \backslash 29-J u n-2003 \backslash 0 \backslash \backslash \#$ B 3LYP/6 


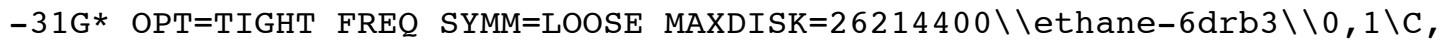
$-0.7652714924,0.0 . \backslash \mathrm{C}, 0.7652714924,0 ., 0 . \backslash \mathrm{H}, 1.1643473724,1.0209355355,0$ $.0000019949 \backslash \mathrm{H}, 1.1643473724,-0.5104694954,0.8841551119 \backslash \mathrm{H}, 1.1643473724,-$ $0.5104660401,-0.8841571069 \backslash \mathrm{H},-1.1643473724,-1.0209355355,-0.0000019949$ $\backslash \mathrm{H},-1.1643473724,0.5104660401,0.8841571069 \backslash \mathrm{H},-1.1643473724,0.510469495$ $4,-0.8841551119 \backslash \backslash$ Version=DEC-AXP-OSF $/ 1-G 03$ RevB . 03 $\backslash$ State $=1-A 1 G \backslash H F=-79.8$ $304175 \backslash \mathrm{RMSD}=4.869 \mathrm{e}-10 \backslash \mathrm{RMSF}=8.778 \mathrm{e}-07 \backslash \mathrm{Dipole}=0,0 ., 0 . \backslash \mathrm{PG}=\mathrm{D} 03 \mathrm{D}[\mathrm{C} 3(\mathrm{C} 1 . \mathrm{C} 1$ )$, 3 \operatorname{SGD}(\mathrm{H} 2)] \backslash \backslash \mathrm{a}$

\section{- $\mathrm{CH}_{2} \mathrm{CH}_{2} \mathrm{Ph}$}

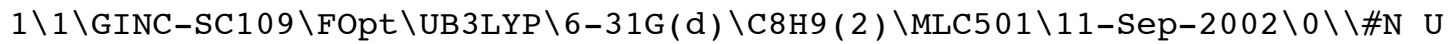
B3LYP $/ 6-31 \mathrm{G}(\mathrm{D}) \quad \mathrm{OPT}=(\mathrm{MAXCYC}=100, \mathrm{TIGHT}) \quad \mathrm{FREQ} \quad \mathrm{SCF}=(\mathrm{TIGHT}, \mathrm{MAXCYC}=100)$ MAXD $I S K=52428800 \backslash \backslash e t b z \_r a d-c 1 a-6 d u b 3 \backslash \backslash 0,2 \backslash C,-2.03452684,-2.0512941126,0.33$ $24478083 \backslash \mathrm{C},-0.6420304454,-1.9399542889,-0.2000743247 \backslash \mathrm{C},-0.0508558132,-$ $0.5429243693,-0.1134582342 \backslash \mathrm{C}, 0.4913398018,0.0842541701,-1.2410059535 \backslash \mathrm{C}$ $,-0.0274801177,0.1457417281,1.1080457068 \backslash C, 1.046074949,1.3631473884,-1$ $.1547761696 \backslash \mathrm{C}, 0.5252915484,1.4226125461,1.1993467209 \backslash \mathrm{C}, 1.0649541968,2$. $036857646,0.0665065029 \backslash \mathrm{H}, 1.4951343558,3.0324663079,0.136287752 \backslash \mathrm{H}, 1.460$ $3795209,1.8325247546,-2.0434783758 \backslash \mathrm{H}, 0.5352032701,1.9393156892,2.15579$ $3481 \backslash \mathrm{H}, 0.4781960201,-0.4336517467,-2.1977738577 \backslash \mathrm{H},-0.449973448,-0.3270$ $655114,1.9916602032 \backslash \mathrm{H}, 0.00765341,-2.6500255972,0.3352581197 \backslash \mathrm{H},-0.60835$ $41106,-2.2704429873,-1.2545284257 \backslash \mathrm{H},-2.4241734132,-3.0089567382,0.6633$ $438848 \backslash \mathrm{H},-2.7306692819,-1.2248084189,0.2312448771 \backslash \backslash$ Version=DEC-AXP-OSF /1-G98RevA. $11.3 \backslash \mathrm{HF}=-310.2072089 \backslash \mathrm{S} 2=0.753928 \backslash \mathrm{S} 2-1=0 . \backslash \mathrm{S} 2 \mathrm{~A}=0.75001 \backslash \mathrm{RMSD}=8$ $.145 \mathrm{e}-09 \backslash \mathrm{RMSF}=1.629 \mathrm{e}-06 \backslash \mathrm{Dipole}=0.0104278,-0.1026318,-0.0496021 \backslash \mathrm{PG}=\mathrm{C} 01$ $[\mathrm{X}(\mathrm{C} 8 \mathrm{H} 9)] \backslash \backslash @$

\section{$\mathrm{H}-\mathrm{CH}_{2} \mathrm{CH}_{2} \mathrm{Ph}$}

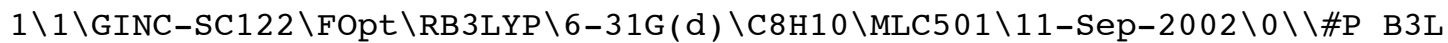
$\mathrm{YP} / 6-31 \mathrm{G}(\mathrm{D}) \mathrm{FOPT}=(\mathrm{MAXCYC}=100)$ FREQ MAXDISK=52428800 SCF=(TIGHT, MAXCYC $=$ $100) \mathrm{GEOM}=\mathrm{CHECK} \backslash \backslash$ etbz $-\mathrm{cs}-6 \mathrm{drb} 3 \backslash \backslash 0,1 \backslash \mathrm{C},-2.2284396309,1.7737516665,0 . \backslash \mathrm{C}$, $-0.6937105063,1.8926699354,0 . \backslash C, 0 ., 0.546181554,0 . \backslash C, 0.3118254669,-0.10$ $23160492,1.2026834225 \backslash \mathrm{C}, 0.3118254669,-0.1023160492,-1.2026834225 \backslash \mathrm{C}, 0.9$ $132220777,-1.3616103083,1.2059190433 \backslash C, 0.9132220777,-1.3616103083,-1.2$ $059190433 \backslash \mathrm{C}, 1.2153222385,-1.9966792384,0 . \backslash \mathrm{H}, 1.6863498799,-2.976105526$, $0 . \backslash \mathrm{H}, 1.150067146,-1.8447129978,2.1505767223 \backslash \mathrm{H}, 1.150067146,-1.844712997$ $8,-2.1505767223 \backslash \mathrm{H}, 0.0837929653,0.387950677,2.1471919348 \backslash \mathrm{H}, 0.0837929653$ $, 0.387950677,-2.1471919348 \backslash \mathrm{H},-0.3779096411,2.4687487917,-0.8791267801 \backslash$ $\mathrm{H},-0.3779096411,2.4687487917,0.8791267801 \backslash \mathrm{H},-2.5794995785,1.2298765659$ $, 0.8842051352 \backslash \mathrm{H},-2.5794995785,1.2298765659,-0.8842051352 \backslash \mathrm{H},-2.69885480$ $66,2.7639522374,0 . \backslash \backslash$ Version=DEC-AXP-OSF / 1-G98RevA.11.3 State=1-A ' $\backslash \mathrm{HF}=-$ $310.8802404 \backslash \mathrm{RMSD}=5.588 \mathrm{e}-09 \backslash \mathrm{RMSF}=2.772 \mathrm{e}-06 \backslash \mathrm{Dipole}=-0.0632775,0.0900445$, $0 . \backslash \mathrm{PG}=\mathrm{CS}[\mathrm{SG}(\mathrm{C} 4 \mathrm{H} 2), \mathrm{X}(\mathrm{C} 4 \mathrm{H} 8)] \backslash \backslash @$

\section{$\cdot \mathrm{CH}_{2} \mathrm{SCH}_{3}$}

$1 \backslash 1 \backslash G I N C-S C 40 \backslash F O p t \backslash U B 3 L Y P \backslash 6-31 G(d) \backslash C 2 H 5 S 1(2) \backslash R O O T \backslash 09-A u g-2001 \backslash 1 \backslash \backslash \# P$ UB 3LYP $/ 6-31 \mathrm{G}$ * GEOM=CHECK GUESS=READ FOPT=Z-MATRIX FREQ MAXDISK=393216000 $0 \backslash \backslash \mathrm{CH} 3-\mathrm{S}-\mathrm{CH} 2 * \operatorname{rad} \mathrm{C} 1 \backslash \backslash 0,2 \backslash \mathrm{S} \backslash \mathrm{C}, 1, \mathrm{Cs} 1 \backslash \mathrm{H}, 2, \mathrm{hc} 1,1, \mathrm{hcs} 1 \backslash \mathrm{H}, 2, \mathrm{hc} 2,1, \mathrm{hcs} 2,3, \mathrm{dh}$ $1,0 \backslash \mathrm{C}, 1, \mathrm{cs} 2,2, \mathrm{csc}, 3, \mathrm{dh} 2,0 \backslash \mathrm{H}, 5, \mathrm{hc} 3,1, \mathrm{hcs} 3,2, \mathrm{dh} 3,0 \backslash \mathrm{H}, 5, \mathrm{hc} 4,1, \mathrm{hcs} 4,2, \mathrm{dh} 4$, $0 \backslash \mathrm{H}, 5$, hc5 1 , hcs 5,2 , dh $5,0 \backslash \backslash \mathrm{cs} 1=1.72792339 \backslash \mathrm{hc} 1=1.08323195 \backslash$ hc $2=1.08486743$ $\backslash \mathrm{cs} 2=1.82757399 \backslash \mathrm{hc} 3=1.09259995 \backslash \mathrm{hc} 4=1.09284674 \backslash \mathrm{hc} 5=1.09379903 \backslash \mathrm{hcs} 1=116$. $54833307 \backslash \mathrm{hcs} 2=120.61117155 \backslash \mathrm{csc}=101.38957243 \backslash \mathrm{hcs} 3=107.21472595 \backslash \mathrm{hcs} 4=110$ $.5393542 \backslash$ hcs $5=110.59047311 \backslash \mathrm{dh} 1=-159.93202602 \backslash \mathrm{dh} 2=-173.68441562 \backslash \mathrm{dh} 3=-17$ $7.97662667 \backslash \mathrm{dh} 4=62.80681521 \backslash \mathrm{dh} 5=-59.18214036 \backslash \backslash$ Version=DEC-AXP-OSF $/ 1-G 98$ RevA. $9 \backslash \mathrm{HF}=-477.3516401 \backslash \mathrm{S} 2=0.754164 \backslash \mathrm{S} 2-1=0 . \backslash \mathrm{S} 2 \mathrm{~A}=0.750013 \backslash \mathrm{RMSD}=3.903 e-09$ $\backslash \mathrm{RMSF}=1.827 \mathrm{e}-05 \backslash \mathrm{Dipole}=-0.5687768,-0.0455824,0.0333926 \backslash \mathrm{PG}=\mathrm{C} 01] \mathrm{X}(\mathrm{C} 2 \mathrm{H} 5 \mathrm{~S}$ $1)] \backslash \backslash @$ 


\section{$\mathrm{H}-\mathrm{CH}_{2} \mathrm{SCH}_{3}$}

$1 \backslash 1 \backslash G I N C-S C 32 \backslash F O p t \backslash R B 3 L Y P \backslash 6-31 G(d) \backslash C 2 H 6 S 1 \backslash M L C 501 \backslash 16-M a r-2004 \backslash 0 \backslash \backslash \#$ B3LY

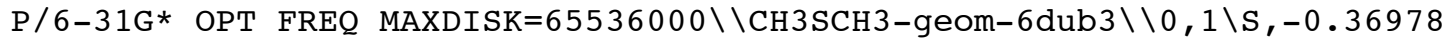
$21769,-0.5514550776,0 . \backslash \mathrm{C}, 1.4449112088,-0.3482525121,0 . \backslash \mathrm{C},-0.8707345203$ $, 1.2045205438,0 . \backslash \mathrm{H}, 1.8814896329,-1.3505755523,0 . \backslash \mathrm{H},-1.9637607463,1.227$ $8848837,0 . \backslash \mathrm{H}, 1.785254922,0.1830801241,0.8945611212 \backslash \mathrm{H},-0.5083920151,1.7$ $21101736,0.8945611186 \backslash \mathrm{H}, 1.785254922,0.1830801241,-0.8945611212 \backslash \mathrm{H},-0.50$ $83920151,1.721101736,-0.8945611186 \backslash \backslash$ Version=DEC-AXP-OSF / 1-G03RevB.03\S tate $=1-A^{\prime} \backslash H F=-478.0138083 \backslash R M S D=4.490 e-09 \backslash R M S F=3.553 e-05 \backslash D i p o l e=0.37734$ $84,0.5627385,0 . \backslash \mathrm{PG}=\mathrm{CS} \quad[\mathrm{SG}(\mathrm{C} 2 \mathrm{H} 2 \mathrm{~S} 1), \mathrm{X}(\mathrm{H} 4)] \backslash \backslash @$

\section{$\cdot \mathrm{CH}_{2} \mathrm{SCH}_{2} \mathrm{Ph}$}

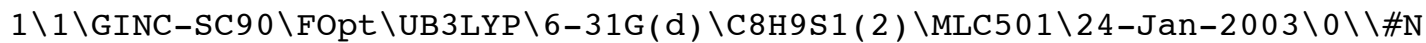
B3LYP/6-31G(D) OPT FREQ MAXDISK=39321600 GEOM=CHECK GUESS=READ \\CH2SB2

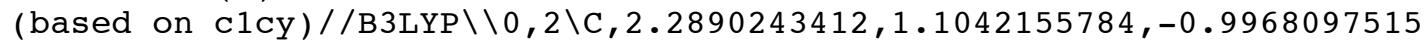
$\backslash \mathrm{S}, 2.4682148589,-0.3907221864,-0.1509036442 \backslash \mathrm{C}, 1.1225379022,-0.28621318$ $16,1.1225476215 \backslash \mathrm{H}, 1.3107207601,1.5610575659,-1.102603258 \backslash \mathrm{H}, 3.075599949$ $2,1.3512283071,-1.7000127755 \backslash C,-0.2711493981,-0.1551745282,0.564326655$ $8 \backslash \mathrm{H}, 1.3608755879,0.5488633714,1.7871423637 \backslash \mathrm{H}, 1.2376766027,-1.214308172$ $2,1.6915234601 \backslash \mathrm{C},-0.95329325,1.0657421787,0.6394616707 \backslash \mathrm{C},-0.9121192978$ $,-1.2480730672,-0.0376886 \backslash \mathrm{C},-2.2464469979,1.1946439378,0.1265252273 \backslash \mathrm{C}$, $-2.2022041354,-1.1226946014,-0.5478815353 \backslash C,-2.8740810671,0.1007283115$ $,-0.4679674561 \backslash \mathrm{H},-3.8807489186,0.1974088184,-0.8655582935 \backslash \mathrm{H},-2.6863783$ $19,-1.9804336061,-1.007245593 \backslash \mathrm{H},-2.7612133853,2.1493477275,0.194971213$ $4 \backslash \mathrm{H},-0.39086816,-2.1998103758,-0.1093106115 \backslash \mathrm{H},-0.4707104422,1.91915357$ $78,1.1104688062 \backslash \backslash$ Version=DEC-AXP-OSF / 1-G98RevA. $11.3 \backslash \mathrm{HF}=-708.4022103 \backslash \mathrm{S} 2$ $=0.754322 \backslash \mathrm{S} 2-1=0 . \backslash \mathrm{S} 2 \mathrm{~A}=0.750014 \backslash \mathrm{RMSD}=3.796 \mathrm{e}-09 \backslash \mathrm{RMSF}=2.003 e-06 \backslash \mathrm{Dipole}=-0$ $.5920659,0.0932405,0.1849989 \backslash \mathrm{PG}=\mathrm{C} 01[\mathrm{X}(\mathrm{C} 8 \mathrm{H} 9 \mathrm{~S} 1)] \backslash \backslash @$

\section{$\mathrm{H}-\mathrm{CH}_{2} \mathrm{SCH}_{2} \mathrm{Ph}$}

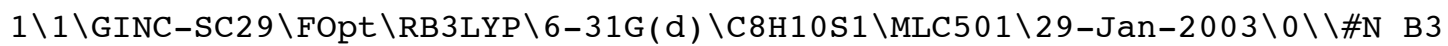
LYP /6-31G(D) OPT (MAXCYC=100) FREQ MAXDISK=39321600 GEOM=CHECK GUESS=RE

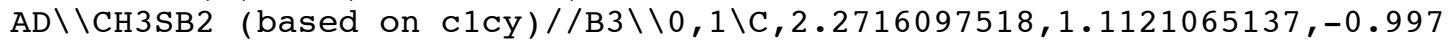
$3390939 \backslash \mathrm{S}, 2.4284127182,-0.4787724374,-0.1153723018 \backslash \mathrm{C}, 1.0853222086,-0.2$ $985429716,1.1422050296 \backslash \mathrm{H}, 1.2732481361,1.2266499171,-1.4278935723 \backslash \mathrm{H}, 3.0$ $083318311,1.0980264415,-1.804834343 \backslash \mathrm{C},-0.3064073801,-0.1601063939,0.57$ $40714721 \backslash \mathrm{H}, 1.3243469567,0.5507382422,1.7912300283 \backslash \mathrm{H}, 1.1749078686,-1.20$ $73821913,1.7469281007 \backslash \mathrm{C},-0.9937221494,1.0564749042,0.6650093261 \backslash \mathrm{C},-0.9$ $397240463,-1.2425042601,-0.0546658397 \backslash \mathrm{C},-2.2834744781,1.1918881437,0.1$ $448090955 \backslash \mathrm{C},-2.2266742838,-1.1118376907,-0.5716986532 \backslash \mathrm{C},-2.9038127115$, $0.1075313971,-0.4741765207 \backslash \mathrm{H},-3.9076158778,0.2087023447,-0.8780254106 \backslash$ $\mathrm{H},-2.7042121176,-1.962380853,-1.0511911286 \backslash \mathrm{H},-2.8009101292,2.144302445$ $6,0.2259204072 \backslash \mathrm{H},-0.4126995505,-2.1894404946,-0.1430459346 \backslash \mathrm{H},-0.517664$ $1431,1.9038244544,1.1539361163 \backslash \mathrm{H}, 2.488962066,1.9572608373,-0.336356329$ $5 \backslash \backslash$ Version=DEC-AXP-OSF / 1-G98RevA. 11.3\HF=-709.0645592 \RMSD=4.540e-09 $\backslash \mathrm{R}$ $\mathrm{MSF}=5.598 \mathrm{e}-06 \backslash \mathrm{Dipole}=-0.4507539,0.4538128,0.0558314 \backslash \mathrm{PG}=\mathrm{C} 01 \quad[\mathrm{X}(\mathrm{C} 8 \mathrm{H} 10 \mathrm{~S} 1)$ ]$\backslash \backslash @$

\section{$\cdot \mathrm{CH}_{2} \mathrm{SCH}_{2} \mathrm{COOCH}_{3}$}

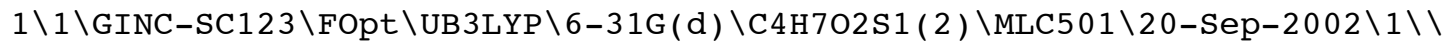
\#N UB3LYP/6-31G(D) OPT=(Z-MATRIX, MAXCYC=100) FREQ MAXDISK=52428800 GEO $\mathrm{M}=\mathrm{CHECK} \backslash \backslash \mathrm{ch} 2 \mathrm{sch} 2 \mathrm{co} 2 \mathrm{ch} 3-\mathrm{c} 1 \mathrm{p}-6 \mathrm{dub} 3 \backslash \backslash 0,2 \backslash \mathrm{S} \backslash \mathrm{C}, 1, \mathrm{~B} 1 \backslash \mathrm{H}, 2, \mathrm{~B} 2,1, \mathrm{~A} 1 \backslash \mathrm{H}, 2, \mathrm{~B} 3,1, \mathrm{~A} 2$ $, 3, \mathrm{D} 1,0 \backslash \mathrm{C}, 1, \mathrm{~B} 4,2, \mathrm{~A} 3,3, \mathrm{D} 2,0 \backslash \mathrm{C}, 5, \mathrm{~B} 5,1, \mathrm{~A} 4,2, \mathrm{D} 3,0 \backslash 0,6, \mathrm{~B} 6,5, \mathrm{~A} 5,1, \mathrm{D} 4,0 \backslash 0,6, \mathrm{~B}$ $7,5, \mathrm{~A} 6,7, \mathrm{D} 5,0 \backslash \mathrm{C}, 8, \mathrm{~B} 8,6, \mathrm{~A} 7,5, \mathrm{D} 6,0 \backslash \mathrm{H}, 5, \mathrm{~B} 9,1, \mathrm{~A} 8,6, \mathrm{D} 7,0 \backslash \mathrm{H}, 5, \mathrm{~B} 10,1, \mathrm{~A} 9,6, \mathrm{D} 8$, $0 \backslash \mathrm{H}, 9, \mathrm{~B} 11,8, \mathrm{~A} 10,6, \mathrm{D} 9, \mathrm{O} \backslash \mathrm{H}, 9, \mathrm{~B} 12,8, \mathrm{~A} 11,12, \mathrm{D} 10,0 \backslash \mathrm{H}, 9, \mathrm{~B} 13,8, \mathrm{~A} 12,12, \mathrm{D} 11,0 \backslash \backslash$ $\mathrm{B} 1=1.73218229 \backslash \mathrm{B} 2=1.08339889 \backslash \mathrm{B} 3=1.08374008 \backslash \mathrm{B} 4=1.84209974 \backslash \mathrm{B} 5=1.51467098 \backslash$ $\mathrm{B} 6=1.21315793 \backslash \mathrm{B} 7=1.34658231 \backslash \mathrm{B} 8=1.43874406 \backslash \mathrm{B} 9=1.09170656 \backslash \mathrm{B} 10=1.09162575$ 
$\backslash \mathrm{B} 11=1.09303436 \backslash \mathrm{B} 12=1.09303538 \backslash \mathrm{B} 13=1.09003054 \backslash \mathrm{A} 1=115.86996819 \backslash \mathrm{A} 2=120.0$ $2408378 \backslash A 3=101.45128476 \backslash A 4=111.37524829 \backslash A 5=124.40766091 \backslash A 6=111.3920199$ $9 \backslash A 7=115.34855438 \backslash A 8=108.87789335 \backslash A 9=105.81007532 \backslash A 10=110.50131068 \backslash A 11$ $=110.36047466 \backslash A 12=105.48128035 \backslash D 1=-159.27059229 \backslash D 2=183.88538086 \backslash D 3=-67$ $.25514346 \backslash D 4=86.64066542 \backslash D 5=-178.52626952 \backslash D 6=176.73687142 \backslash D 7=120.66718$ $891 \backslash D 8=-120.75782314 \backslash D 9=60.16144943 \backslash D 10=-120.73140431 \backslash D 11=119.69454139$ $\backslash \backslash$ Version=DEC-AXP-OSF /1-G98RevA. $11.3 \backslash \mathrm{HF}=-705.2213851 \backslash \mathrm{S} 2=0.754133 \backslash \mathrm{S} 2-1=$ $0 . \backslash \mathrm{S} 2 \mathrm{~A}=0.750013 \backslash \mathrm{RMSD}=5.196 \mathrm{e}-09 \backslash \mathrm{RMSF}=3.766 \mathrm{e}-05 \backslash \mathrm{Dipole}=-0.320091,-0.3251$ $822,-0.5102612 \backslash \mathrm{PG}=\mathrm{C} 01 \quad[\mathrm{X}(\mathrm{C} 4 \mathrm{H} 7 \mathrm{O} 2 \mathrm{~S} 1)] \backslash \backslash @$

\section{$\mathrm{H}-\mathrm{CH}_{2} \mathrm{SCH}_{2} \mathrm{COOCH}_{3}$}

$1 \backslash 1 \backslash G I N C-S C 28 \backslash F O p t \backslash R B 3 L Y P \backslash 6-31 G(d) \backslash C 4 H 8 O 2 S 1 \backslash M L C 501 \backslash 20-S e p-2002 \backslash 1 \backslash \backslash \# N \quad B$ 3LYP/6-31G(D) OPT=(Z-MATRIX, MAXCYC=100) FREQ MAXDISK=52428800 GEOM=CHE $\mathrm{CK} \backslash \backslash \mathrm{ch} 3 \mathrm{sch} 2 \mathrm{co} 2 \mathrm{ch} 3-\mathrm{c} 1 \mathrm{p}-6 \mathrm{dub} 3 \backslash \backslash 0,1 \backslash \mathrm{S} \backslash \mathrm{C}, 1, \mathrm{~B} 1 \backslash \mathrm{H}, 2, \mathrm{~B} 2,1, \mathrm{~A} 1 \backslash \mathrm{H}, 2, \mathrm{~B} 3,1, \mathrm{~A} 2,3, \mathrm{D} 1$ , $0 \backslash \mathrm{C}, 1, \mathrm{~B} 4,2, \mathrm{~A} 3,3, \mathrm{D} 2,0 \backslash \mathrm{C}, 5, \mathrm{~B} 5,1, \mathrm{~A} 4,2, \mathrm{D} 3,0 \backslash 0,6, \mathrm{~B} 6,5, \mathrm{~A} 5,1, \mathrm{D} 4,0 \backslash 0,6, \mathrm{~B} 7,5, \mathrm{~A}$ $6,7, \mathrm{D} 5,0 \backslash \mathrm{C}, 8, \mathrm{~B} 8,6, \mathrm{~A} 7,5, \mathrm{D} 6,0 \backslash \mathrm{H}, 5, \mathrm{~B} 9,1, \mathrm{~A} 8,6, \mathrm{D} 7,0 \backslash \mathrm{H}, 5, \mathrm{~B} 10,1, \mathrm{~A} 9,6, \mathrm{D} 8,0 \backslash \mathrm{H}, 9$ , $\mathrm{B} 11,8, \mathrm{~A} 10,6, \mathrm{D} 9,0 \backslash \mathrm{H}, 9$, $\mathrm{B} 12,8, \mathrm{~A} 11,12, \mathrm{D} 10,0 \backslash \mathrm{H}, 9, \mathrm{~B} 13,8, \mathrm{~A} 12,12, \mathrm{D} 11,0 \backslash \mathrm{H}, 2$, $\mathrm{B} 1$ $4,1, \mathrm{~A} 13,4, \mathrm{D} 12,0 \backslash \backslash \mathrm{B} 14=1.09407907 \backslash \mathrm{A} 13=110.98917864 \backslash \mathrm{D} 12=-122.32599148 \backslash \mathrm{B} 1=$ $1.82941218 \backslash \mathrm{B} 2=1.09320983 \backslash \mathrm{B} 3=1.09179061 \backslash \mathrm{B} 4=1.84120939 \backslash \mathrm{B} 5=1.51326922 \backslash \mathrm{B} 6=$ $1.21430186 \backslash B 7=1.34788275 \backslash B 8=1.43778776 \backslash B 9=1.09325654 \backslash B 10=1.09174033 \backslash B 1$ $1=1.09310389 \backslash \mathrm{B} 12=1.09323525 \backslash \mathrm{B} 13=1.09017352 \backslash \mathrm{A} 1=106.45059106 \backslash \mathrm{A} 2=110.8007$ $717 \backslash \mathrm{A} 3=100.17137515 \backslash \mathrm{A} 4=111.04905442 \backslash \mathrm{A} 5=124.57420924 \backslash \mathrm{A} 6=111.47577278 \backslash \mathrm{A} 7$ $=115.34918093 \backslash \mathrm{A} 8=109.61040514 \backslash \mathrm{A} 9=106.06955231 \backslash \mathrm{A} 10=110.56850432 \backslash \mathrm{A} 11=110$ $.39708436 \backslash A 12=105.51426102 \backslash D 1=-119.0645737 \backslash D 2=175.11314455 \backslash D 3=-70.7090$ $079 \backslash D 4=81.86780755 \backslash D 5=-178.35716699 \backslash D 6=176.4774588 \backslash D 7=120.89987776 \backslash D 8=$ $-120.6551676 \backslash D 9=59.44174906 \backslash D 10=-120.72743755 \backslash D 11=119.73808134 \backslash \backslash$ Versio $\mathrm{n}=\mathrm{DEC}-\mathrm{AXP}-\mathrm{OSF} / 1-\mathrm{G} 98 \mathrm{RevA} .11 .3 \backslash \mathrm{HF}=-705.8848169 \backslash \mathrm{RMSD}=7.903 \mathrm{e}-09 \backslash \mathrm{RMSF}=2.103$

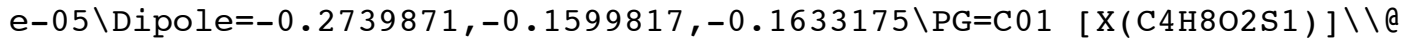

\section{$\cdot \mathrm{CH}_{2} \mathrm{SC}\left(\mathrm{CH}_{3}\right)_{2} \mathrm{CN}$}

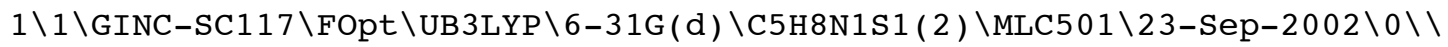
\#N UB3LYP/6-31G(D) OPT= $(M A X C Y C=100)$ FREQ MAXDISK=52428800 GEOM=CHECK \\

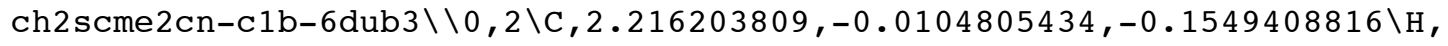
$2.2124891486,-0.0104815977,0.9305891576 \backslash \mathrm{H}, 3.1568671101,-0.0197233775,-$ $0.6925761048 \backslash \mathrm{S}, 0.8286229278,-0.5339169106,-1.0366526174 \backslash \mathrm{C},-0.599969057$ $1,0.1901463328,-0.0522060574 \backslash \mathrm{C},-0.5915749507,1.7270808565,-0.146789857$ $3 \backslash \mathrm{C},-1.8833176522,-0.4115520613,-0.6559343429 \backslash \mathrm{C},-0.4582679961,-0.22442$ $8565,1.3499281472 \backslash \mathrm{H}, 0.3497812116,2.1362691642,0.2283017181 \backslash \mathrm{H},-0.712347$ $3903,2.0280941129,-1.1915585968 \backslash \mathrm{H},-1.4165004748,2.1450622339,0.4414051$ $552 \backslash \mathrm{H},-1.9564943352,-0.1369539714,-1.7131055672 \backslash \mathrm{H},-2.7597863423,-0.015$ $4848027,-0.133271841 \backslash \mathrm{H},-1.8901665774,-1.5015087304,-0.5724657191 \backslash \mathrm{N},-0$. $3343220162,-0.5295997971,2.4641116613 \backslash \backslash$ Version=DEC-AXP-OSF / 1-G98RevA. 1 $1.3 \backslash \mathrm{HF}=-648.2151962 \backslash \mathrm{S} 2=0.755015 \backslash \mathrm{S} 2-1=0 . \backslash \mathrm{S} 2 \mathrm{~A}=0.750017 \backslash \mathrm{RMSD}=7.231 \mathrm{e}-09 \backslash \mathrm{RM}$ $\mathrm{SF}=2.917 \mathrm{e}-06 \backslash \mathrm{Dipole}=-0.3235252,0.5904565,-1.0178268 \backslash \mathrm{PG}=\mathrm{C} 01 \quad[\mathrm{X}(\mathrm{C} 5 \mathrm{H} 8 \mathrm{~N} 1 \mathrm{~S} 1$ ) $] \backslash \backslash$ Q

\section{$\mathrm{H}-\mathrm{CH}_{2} \mathrm{SC}\left(\mathrm{CH}_{3}\right)_{2} \mathrm{CN}$}

$1 \backslash 1 \backslash G I N C-S C 24 \backslash F O p t \backslash R B 3 L Y P \backslash 6-31 G(d) \backslash C 5 H 9 N 1 S 1 \backslash M L C 501 \backslash 23-$ Sep-2002 $0 \backslash \backslash \# N \quad B$ 3LYP/6-31G(D) OPT $=($ MAXCYC=100) FREQ MAXDISK=52428800 GEOM=CHECK $\backslash \mathrm{ch} 3 \mathrm{sC}$

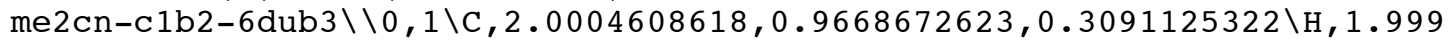
$9993174,0.9636095319,1.402206942 \backslash \mathrm{H}, 3.036058107,0.9668243234,-0.0412801$ $942 \backslash \mathrm{S}, 1.2416814904,-0.5438703598,-0.3838305371 \backslash \mathrm{C},-0.5503501855,-0.3539$ $747574,0.1076597413 \backslash \mathrm{C},-0.7388086095,-0.429265126,1.635054428 \backslash \mathrm{C},-1.3023$ $225301,-1.501267512,-0.5974700784 \backslash \mathrm{C},-1.035494258,0.9424549998,-0.38849$ $77241 \backslash \mathrm{H},-0.3703085291,-1.3927528237,1.9987089375 \backslash \mathrm{H},-0.1981988796,0.370$ $0798125,2.1489303456 \backslash \mathrm{H},-1.8009991951,-0.3359079691,1.8893357496 \backslash \mathrm{H},-1.1$ $916771354,-1.4397566369,-1.6831554124 \backslash \mathrm{H},-2.3679363761,-1.4558307861,-0$ $.3504707881 \backslash \mathrm{H},-0.906511597,-2.4628171846,-0.2554545166 \backslash \mathrm{N},-1.4011523275$ 
$, 1.9824019672,-0.7563977594 \backslash \mathrm{H}, 1.4998250629,1.8627745195,-0.0679015481 \backslash$

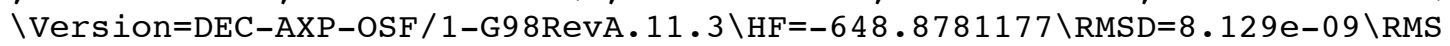
$\mathrm{F}=4.157 \mathrm{e}-06 \backslash \mathrm{Dipole}=0.2984912,-0.8362157,0.8124852 \backslash \mathrm{PG}=\mathrm{C} 01 \quad[\mathrm{X}(\mathrm{C} 5 \mathrm{H} 9 \mathrm{~N} 1 \mathrm{~S} 1)]$ $\backslash \backslash \mathrm{a}$ 